\title{
Magnetic field and radiative transfer modelling of a quiescent prominence $^{\star}$
}

\author{
S. Gunár ${ }^{1}$, P. Schwartz ${ }^{3,2}$, J. Dudík ${ }^{4,5}$, B. Schmieder ${ }^{6}$, P. Heinzel $^{2}$, and J. Jurčák ${ }^{2}$ \\ 1 School of Mathematics and Statistics, University of St Andrews, North Haugh, St Andrews, KY16 9SS, UK \\ e-mail: sg207@st-andrews.ac.uk \\ 2 Astronomical Institute, Academy of Sciences of the Czech Republic, 25165 Ondřejov, Czech Republic \\ 3 Astronomical Institute of the Slovak Academy of Sciences, 05960 Tatranská Lomnica, Slovakia \\ ${ }^{4}$ Department of Applied Mathematics and Theoretical Physics, Centre for Mathematical Sciences, University of Cambridge, \\ Cambridge CB3 0WA, UK \\ 5 Department of Astronomy, Physics of the Earth and Meteorology, Faculty of Mathematics, Physics and Computer Science, \\ Comenius University, Mlynská Dolina F2, 84248 Bratislava, Slovakia \\ ${ }^{6}$ LESIA, Observatoire de Paris, CNRS, UPMC, Univ. Paris Diderot, 5 place Jules Janssen, 92190 Meudon, France
}

Received 2 October 2013 / Accepted 20 May 2014

\section{ABSTRACT}

\begin{abstract}
Aims. The aim of this work is to analyse the multi-instrument observations of the June 22, 2010 prominence to study its structure in detail, including the prominence-corona transition region and the dark bubble located below the prominence body.

Methods. We combined results of the 3D magnetic field modelling with 2D prominence fine structure radiative transfer models to fully exploit the available observations.

Results. The 3D linear force-free field model with the unsheared bipole reproduces the morphology of the analysed prominence reasonably well, thus providing useful information about its magnetic field configuration and the location of the magnetic dips. The 2D models of the prominence fine structures provide a good representation of the local plasma configuration in the region dominated by the quasi-vertical threads. However, the low observed Lyman- $\alpha$ central intensities and the morphology of the analysed prominence suggest that its upper central part is not directly illuminated from the solar surface.

Conclusions. This multi-disciplinary prominence study allows us to argue that a large part of the prominence-corona transition region plasma can be located inside the magnetic dips in small-scale features that surround the cool prominence material located in the dip centre. We also argue that the dark prominence bubbles can be formed because of perturbations of the prominence magnetic field by parasitic bipoles, causing them to be devoid of the magnetic dips. Magnetic dips, however, form thin layers that surround these bubbles, which might explain the occurrence of the cool prominence material in the lines of sight intersecting the prominence bubbles.
\end{abstract}

Key words. Sun: filaments, prominences - magnetic fields - radiative transfer

\section{Introduction}

Prominence observations obtained during campaigns coordinating multiple space-borne and ground-based observatories are relatively rare, but significantly contribute to our understanding of the solar quiescent prominences. Therefore, the data of such well-observed prominences are exploited by many authors who focus their studies on different aspects of each prominence. This is the case of the June 22, 2010 quiescent polar-crown prominence analysed in this paper. It was observed passing over the NW solar limb on June 22,2010 at $56^{\circ} \mathrm{N}$ and $90^{\circ} \mathrm{W}$ by the Solar and Heliospheric Observatory (SOHO), together with the Hinode satellite (Kosugi et al. 2007), the Solar Dynamics Observatory (SDO), and several ground-based observatories.

The dynamical behaviour of this prominence was studied by Berger et al. (2011), who described the evolution of bubbles located below the prominence body that produced small-scale rising plumes. Such dynamical bubbles and plumes were also reported in polar-crown prominences by Berger et al. (2010). The magnetic topology of the prominence bubbles was studied by Dudík et al. (2012), and the possible mechanism triggering the

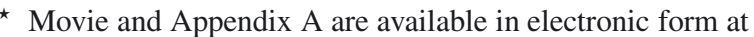
http: //www . aanda.org
}

dynamical behaviour of bubbles and plumes was modelled by Hillier et al. (2012a,b). We here examine several aspects of the prominence bubbles and discuss their possible nature.

One of the fundamental and still open questions about prominences is the nature and the structure of the prominence-corona transition region (PCTR). It is still not clear whether the PCTR surrounds individual prominence fine structures, envelopes the whole prominence in the form of a global shell, consists of multiple PCTR fine structures, or is a combination of these forms. The emission produced by the PCTR was investigated by Parenti et al. (2012) for the June 22, 2010 prominence. Here we attempt to illuminate more details of the PCTR structure.

Up-to-date reviews of the prominence physics can be found in Labrosse et al. (2010) and Mackay et al. (2010), and also in the review of the prominence fine structures by Heinzel (2007) and in the review of their modelling by Gunár (2014). To fully exploit the wealth of available observational data we here combine the magnetic field and radiative transfer modelling with the direct data analyses.

To obtain a better understanding of the magnetic field configuration of this prominence we employ the 3D linear force-free prominence magnetic field model constructed by Dudík et al. (2012). This model is based on the 3D linear force-free model 
of Aulanier \& Demoulin (1998), who have shown that many observed features of filaments, such as their morphology and barbs, may be explained through the location of dips in the twisted magnetic flux ropes (see also Dudík et al. 2008). For more details on the prominence magnetic field modelling, see the reviews by Mackay et al. (2010) and van Ballegooijen \& Su (2014).

Sophisticated models with complex non-LTE (i.e. departures from local thermodynamic equilibrium) radiative transfer computations are needed to properly interpret the available Lyman- $\alpha$ raster observations obtained by the Solar Ultraviolet Measurements of Emitted Radiation (SUMER) spectrograph (Wilhelm et al. 1995) on-board SOHO. Here we use the 2D prominence fine-structure models developed by Heinzel \& Anzer (2001), which represent individual prominence finestructure 2D threads in magneto-hydrostatic (MHS) equilibrium and employ 2D multi-level non-LTE radiative-transfer computations to derive the emerging synthetic hydrogen spectra. These models, in a multi-thread configuration with randomly distributed LOS velocities of individual threads (Gunár et al. 2008), were shown by Gunár et al. (2010) to produce synthetic Lyman spectra that agree well with observations (see also the review by Gunár 2014). The Lyman- $\alpha$ raster observations of quiescent prominences are rare but, because of the very large optical thickness of the Lyman- $\alpha$ line, represent a significant complement to the optically thin $\mathrm{H} \alpha$ observations. The SOHO/SUMER Lyman- $\alpha$ raster prominence observations were previously analysed by for example Gunár et al. (2006). The Lyman spectra including the Lyman- $\alpha$ line were also compared with synthetic spectra obtained by $2 \mathrm{D}$ prominence fine-structure models by Gunár et al. (2007, 2008, 2010).

The present paper is organized in the following way: in Sect. 2 we describe the observed data in detail. In Sect. 3 we present the analysis of the $\mathrm{H} \alpha$ line centre optical thickness. Section 4 gives the details and results of the 3D prominence magnetic field model. In Sect. 5 we analyse the Lyman- $\alpha$ data using the radiative transfer models. Section 6 contains the discussion of the PCTR structure and the nature of the prominence bubble, and in Sect. 7 we give our conclusions.

\section{Observations}

We used the Lyman- $\alpha$ raster observations obtained by the SOHO/SUMER, together with the high-resolution observations in the $\mathrm{H} \alpha$ and $\mathrm{Ca}$ II $\mathrm{H}$ line obtained by the Solar Optical Telescope (SOT, Tsuneta et al. 2008) and the observations obtained by the X-Ray Telescope (XRT, Golub et al. 2007), both on-board Hinode. We also used the full-disk observations by the Atmospheric Imaging Assembly (AIA, Lemen et al. 2012) onboard SDO and the synoptic ground-based observations. In the following subsections we describe the observed data in detail.

\subsection{Ground-based Ho observations}

Synoptic ground-based $\mathrm{H} \alpha$ observations from the Meudon spectroheliograph at the Observatoire de Paris-Meudon and the National Solar Telescope at the Big Bear Solar Observatory (Goode et al. 2003) allow us to see the large-scale structure of the prominence and also of the associated filament occurring prior to the prominence. On-disk observations of this filament obtained several days before June 22, 2010 (Fig. 1, middle) show a string of small absorption structures that most likely represent feet or barbs of a filament, while the filament spine is not visible. This filament crossed the solar limb on June 21 (Fig. 1, bottom) and 22, 2010 (Fig. 1, top). This appearance is typical
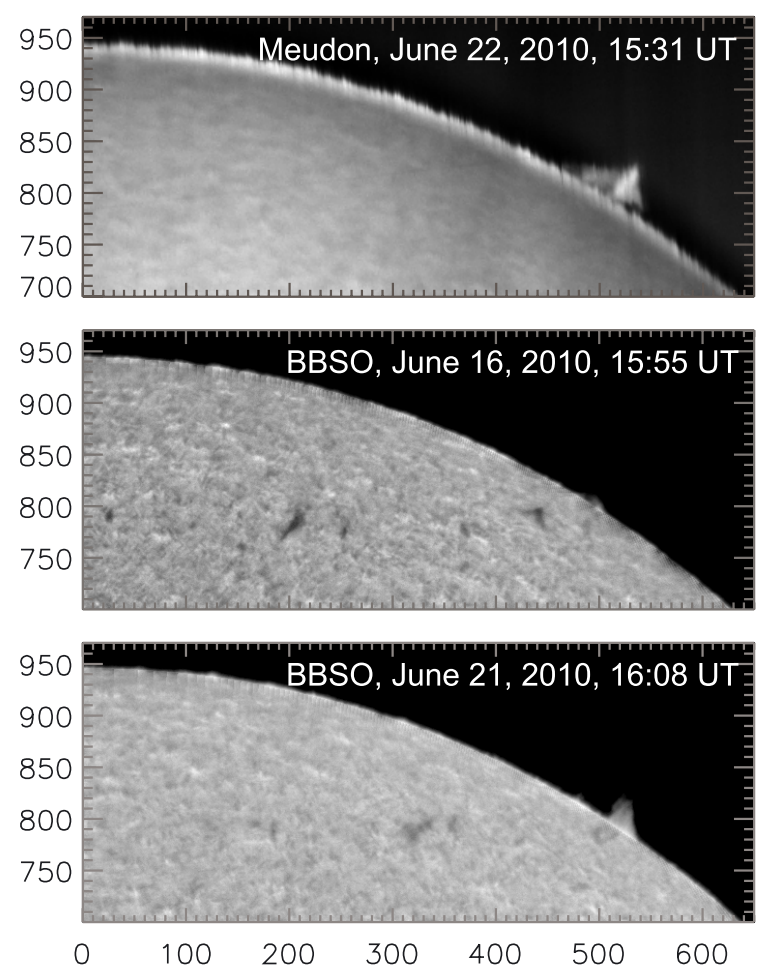

Fig. 1. Synoptic $\mathrm{H} \alpha$ observations from Meudon spectroheliograph (top) and the National Solar Telescope at the BBSO (bottom two) of a weak polar-crown filament that crossed the solar limb on June 21 and 22, 2010. Axes give the solar $X$ and $Y$ coordinates in arcsec.

for the polar-crown filaments during periods of low solar activity observed at high latitudes. This type of polar-crown filament and associated prominence usually does not appear during high solar activity phases.

\subsection{Hinode/SOT}

Hinode/SOT observed this prominence on June 22, 2010 during two time intervals, 15:01-16:06 UT, and 16:40-17:45 UT. The Broadband Filter Imager (BFI) was used for the Ca II H line (the filter is centred on $3968.5 \AA$ and has a band width of $3 \AA$ ) and the Narrowband Filter Imager (NFI) observed at two wavelengths in the blue and red wings of the $\mathrm{H} \alpha$ line $(0.208 \AA$ from the line centre at $6562.8 \AA$ ). The cadence of the BFI and NFI imaging was $14 \mathrm{~s}$. The spatial sampling is $0.11^{\prime \prime}$ and $0.16^{\prime \prime}$ for the BFI and NFI data, which corresponds to a resulting spatial resolution of $0.22^{\prime \prime}$ and $0.32^{\prime \prime}$. Figure 2 shows the prominence observed at 15:02 UT in Ca II H (top) and $\mathrm{H} \alpha$ red wing (middle) with the field of view of $103^{\prime \prime} \times 99^{\prime \prime}$.

These high-resolution observations reveal a large amount of the prominence fine structures. The central part of this prominence is dominated by a bright apparently vertical region surrounded on the left by an extended region filled with numerous quasi-vertical threads. On the right we see more tangled structures with a small bubble located below (between 550"-565" in solar $X$ and $775^{\prime \prime}-790^{\prime \prime}$ in solar $Y$ ). This small bubble is also visible in Fig. 1 (top) and is analysed in this work. A second bubble that is not considered in this paper is located in the far left corner between $490^{\prime \prime}-510^{\prime \prime}$ in solar $X$ and $815^{\prime \prime}-825^{\prime \prime}$ in solar $Y$. These bubbles give rise to frequent dynamical plumes that propagate upwards through the prominence body, as shown for this prominence by Berger et al. (2011). In a detailed inspection 

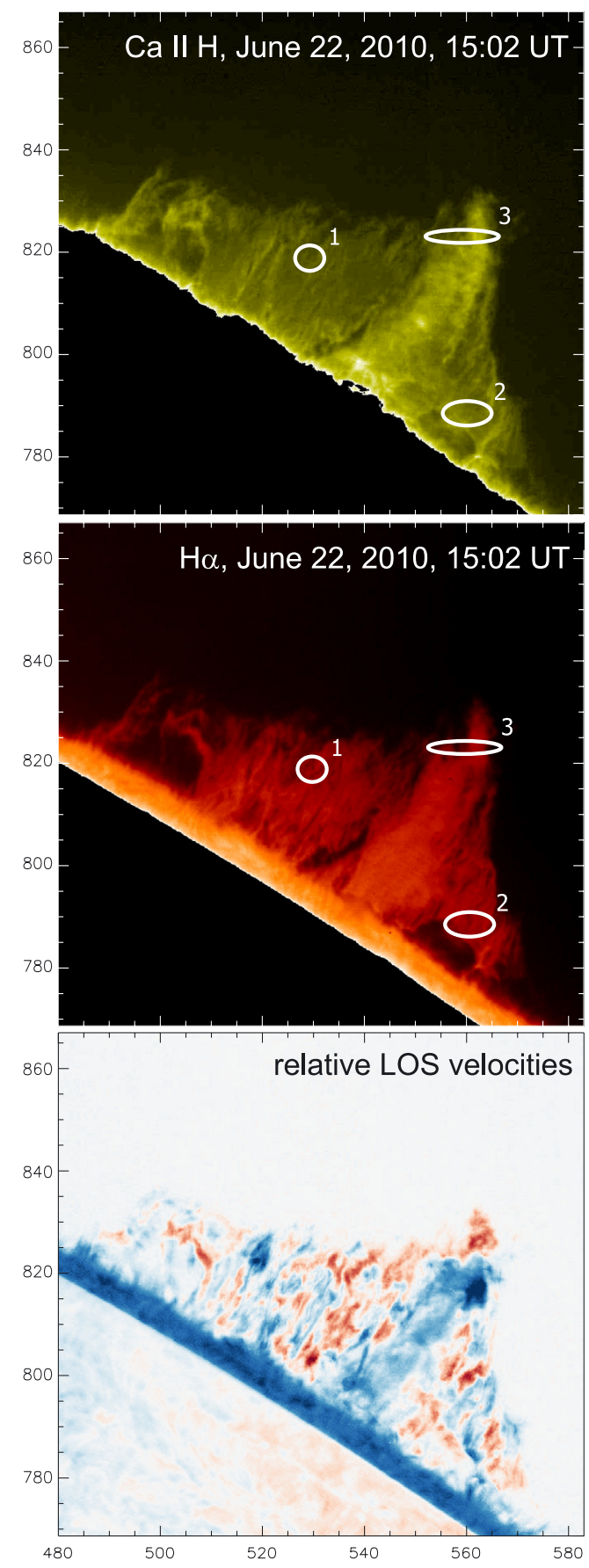

Fig. 2. Hinode/SOT observations of the June 22, 2010 prominence in the Ca II H line (top) and in the red wing $(0.208 \AA$ from the line centre) of the $\mathrm{H} \alpha$ line (middle) at 15:02 UT. Images are aligned to show the same field of view of $103^{\prime \prime} \times 99^{\prime \prime}$. In the bottom panel we display the Dopplergram showing relative LOS velocities obtained from the Hinode/SOT H $\alpha$ line-wing observations. Blue represents the movement towards and red the movement away from the observer. The absolute values of the LOS velocities cannot be determined. Axes give the solar $X$ and $Y$ coordinates in arcsec. White ellipses indicate areas 1, 2, and 3.

the Ca II H image (Fig. 2, top) shows the prominence fine structures with greater detail than the $\mathrm{H} \alpha$ image (Fig. 2, middle), even though in both cases we see the cool prominence plasma with a temperature below $10000 \mathrm{~K}$. However, the optical thickness of these two lines is significantly different. While the typical optical thickness at the $\mathrm{Ca}$ II $\mathrm{H}$ line centre in quiescent prominences is of the order of 10 (based on the experience of authors with 1D prominence modelling, such as Gouttebroze \& Heinzel 2002), the optical thickness of the $\mathrm{H} \alpha$ line in the near wing is lower than unity (based on the experience of authors with 1D prominence modelling, such as Gouttebroze et al. 1993, and with 2D modelling, such as Gunár et al. 2012). This means that the $\mathrm{H} \alpha$ line the emissivity is integrated along the LOS with contribution from all fine structures crossed by the given LOS, which lowers the contrast of prominence fine-structure details. On the other hand, the $\mathrm{Ca}$ II $\mathrm{H}$ line reveals more details of the fine structures in the forefront because of its higher optical thickness. This effect is also enhanced by the slightly higher resolution of the BFI compared to the NFI.

The underlying bubbles are better visible in the $\mathrm{H} \alpha$ line where they appear as dark voids, while in the Ca II H line we can also see some contribution at their location, because of its higher optical thickness. This implies that there is of the cool prominence material somewhere along the LOS traversing them, in front of, behind, or inside the actual bubbles.

For the more detailed analysis of the Lyman- $\alpha$ emission we selected three areas indicated here in Fig. 2. They correspond to distinct morphological features of the June 22, 2010 prominence, with AREA1 placed in a region of numerous quasi-vertical finestructure threads, AREA2 located in the dynamical region just above the bubble, and AREA3 lying in the uppermost part of the central vertical region.

The blue and red wing $\mathrm{H} \alpha$ line observations also allow us to obtain some information on the dynamics of this prominence in addition to the results of Berger et al. (2011). From the $\mathrm{H} \alpha$ intensity maps we can construct the dopplergram using the equation

$v_{\mathrm{DG}}=\left(I_{R}-I_{B}\right) /\left(I_{R}+I_{B}\right)$.

We note that the available $\mathrm{H} \alpha$ line observations were obtained only at two wavelengths within the line profile and lack an absolute intensity calibration. Therefore, the resulting $v_{\mathrm{DG}}$ values are not the real plasma velocities, but merely indicate the direction and relative magnitude of the LOS velocities. The zero LOS velocity was derived using the fact that the left and right wing $\mathrm{H} \alpha$ observations were made at the same distance from the line centre. In Fig. 2 (bottom), we show the resulting Dopplergram, where red and blue correspond to the plasma moving away and towards the observer, respectively. This Dopplergram reveals a fragmented velocity pattern with many small-scale flows with opposite directions (e.g. Zirker et al. 1998). However, it also shows larger coherent motions similar to those studied by Schmieder et al. (2010) and Gunár et al. (2012). Interestingly, the main features of the studied prominence are easily identifiable here as well. The central part is visible as a coherent region moving towards the observer. Numerous quasi-vertical fine threads are also well visible in the LOS velocity pattern. Furthermore, we are also able to identify the bubbles as regions with a very low Doppler signal.

\section{3. $S D O / A I A$}

SDO/AIA images the full Sun with a nominal spatial resolution of $0.6^{\prime \prime}$ and a cadence of up to $12 \mathrm{~s}$ using ten different filters. Seven of these filters are centred on the extremeultraviolet (EUV) wavelengths and are designed to image the solar corona at different plasma temperatures. In this analysis, we used the data obtained with the SDO/AIA 171, 193, and 304 channels between 15:37:50 and 15:37:52 UT that were reduced and co-aligned using standard SolarSoft procedures (details of the data calibration can be found e.g. in Boerner et al. 2012). 

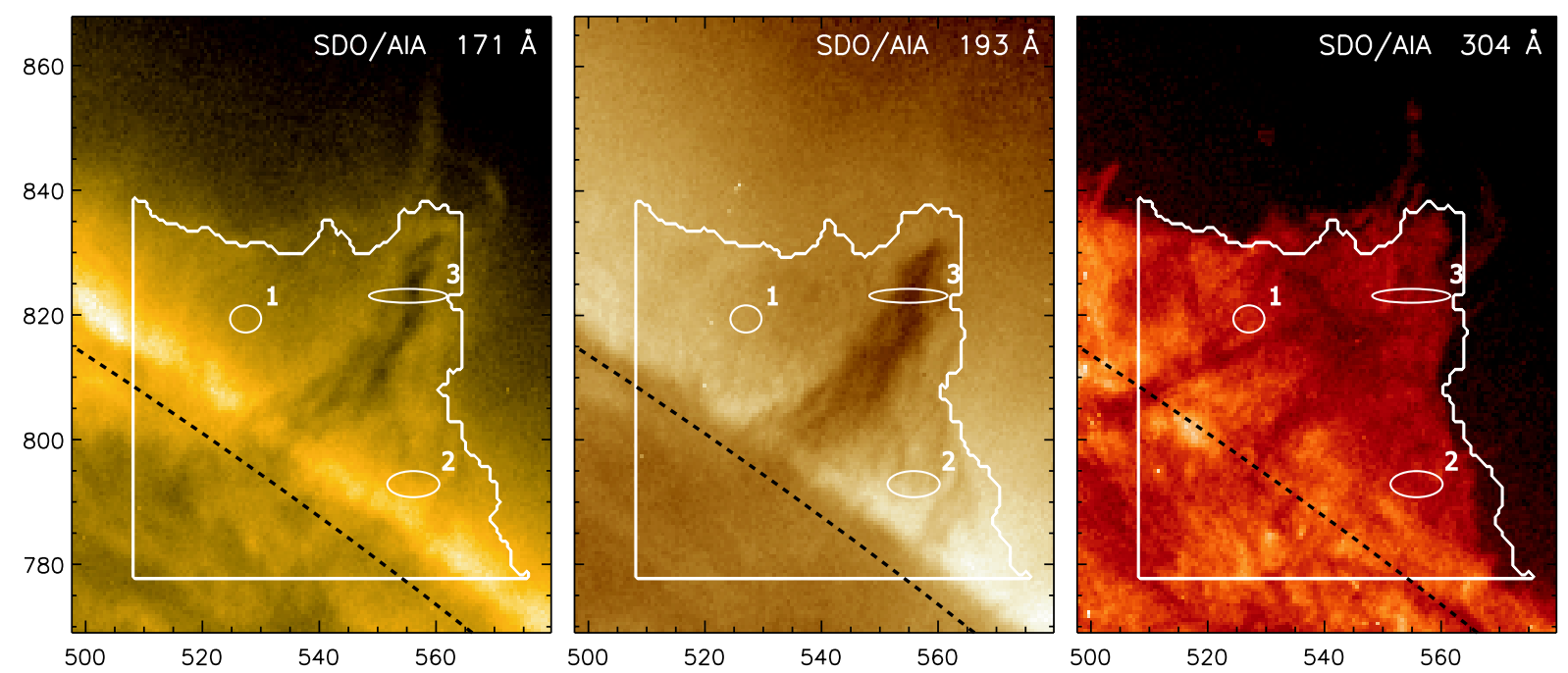

Fig. 3. SDO/AIA 171, 193, and 304 channel observations obtained between 15:37:50 and 15:37:52 UT. White contours outline the edge of the prominence in the Lyman- $\alpha$ raster and dashed lines represent the position of the solar limb. Axes give the solar $X$ and $Y$ coordinates in arcsec. White ellipses indicate areas 1,2, and 3. Temporal evolution in the SDO/AIA 193 and 304 channels in a larger field of view is shown in a movie available online.

Table 1. List of input parameters of the 2D prominence fine structure model MODEL3_6000.

\begin{tabular}{ll}
\hline \hline Model label & Set of input parameters \\
\hline \multirow{3}{*}{ ModEL3_6000 } & $T_{0}=6000 \mathrm{~K} ; T_{\mathrm{tr}}=10^{5} \mathrm{~K} ; \gamma_{1}=10 ; \gamma_{2}=60$ \\
& $B_{x}(0)=5$ gauss $M_{0}=5 \times 10^{-5} \mathrm{~g} \mathrm{~cm}^{-2} ;$ \\
& $p_{\mathrm{tr}}=0.015 \mathrm{dyn} \mathrm{cm}{ }^{-2} ; p_{\mathrm{cen}}=0.13 \mathrm{dyn} \mathrm{cm}^{-2}$ \\
\hline
\end{tabular}

Notes. $T_{0}$ represents the central minimum temperature; $T_{\text {tr }}$ is the boundary transition-region temperature; $\gamma_{1}$ is the exponent representing the shallower temperature gradient along the field; $\gamma_{2}$ is the exponent prescribing the steep temperature gradient in the direction across the magnetic field lines; $B_{x}(0)$ is the magnetic field strength in the middle of the thread; $M_{0}$ gives the maximum column density in the middle of the thread; $p_{\text {tr }}$ represents the boundary gas pressure; and $p_{\text {cen }}$ is the central gas pressure derived from the MHS equilibrium.

The SDO/AIA 171 and 193 show a column-like absorption structure located in the centre of the prominence, oriented nearly vertically with respect to the local solar limb (Fig. 3). This absorption structure corresponds to the similarly shaped brightest region in the Hinode/SOT $\mathrm{Ca} \mathrm{II} \mathrm{H}$ and the $\mathrm{H} \alpha$ line wing images (Fig. 2). The signal in the SDO/AIA 171 channel is dominated by the Fe IX 171.07 $\AA$ line (O'Dwyer et al. 2010; Del Zanna et al. 2011) formed typically at $\log T[\mathrm{~K}]=5.9$. However, under the PCTR conditions, this line can be formed at a lower temperature of $\log T[\mathrm{~K}] \gtrsim 5.6$ (around $400000 \mathrm{~K}$, Parenti et al. 2012). This is responsible for the diffuse emission surrounding the central absorption region that outlines the general shape of the prominence. The extent of the SDO/AIA 171 emission region in the plane-of-the-sky is in many details similar to the extent seen in the SDO/AIA 304 channel. Weak horns (Schmit \& Gibson 2013; Schmit et al. 2013) extending upwards are distinguishable above the main column in the SDO/AIA 171 and 304 channels.

The SDO/AIA 193 channel emission is highly multi-thermal $(\log T[\mathrm{~K}]$ between 6.1 and 7.3) and consists of contributions from several coronal lines of Fe XI and Fe XII (for non-flaring conditions, Del Zanna et al. 2011) with the total contribution by the PCTR lines of $\mathrm{OV}$ and Fe VIII below 2\% (estimated from Table 1 of Parenti et al. 2012). In this channel, the prominence is visible purely in absorption caused by the hydrogen and helium continuum opacity. Moreover, the emissivity-blocking effect (Anzer \& Heinzel 2005) also seems to play a role, as we discuss in Sect. 3. The optical thickness of the photoionization continua at these wavelengths is comparable to the optical thickness in the $\mathrm{H} \alpha$ line (Anzer \& Heinzel 2005), which is around unity.

In the SDO/AIA 304 channel the prominence is observed in emission. This channel is dominated by the He II $303.78 \AA$ line (O'Dwyer et al. 2010), formed mainly by scattering on ionized helium He II (Labrosse \& Gouttebroze 2001; Andretta et al. 2003; Labrosse et al. 2010) created at temperatures around $\log T[\mathrm{~K}] \approx 4.7$ (around $50000 \mathrm{~K}$ ). This line is strongly affected by the opacity effects, similarly to the hydrogen Lyman- $\alpha$ line. Prominences observed in the He II $303.78 \AA$ line look significantly different from those observed in the $\mathrm{H} \alpha$ line, as pointed out by Wang et al. (1998). This is because of high optical thickness of the He II $303.78 \AA$ line which is of the order of $10^{2}-10^{3}$ (Labrosse 2014, priv. comm.). This allows us to observe the structures located on the front side of the prominence. It also helps us to see the parts of the prominence that are too weak to be visible for instance in the $\mathrm{H} \alpha$ line, which renders the prominence in the He II $303.78 \AA$ line emission spatially more extended. In our case, several elongated dark areas are visible as well, but none of them corresponds to the central absorption region visible in the SDO/AIA 171 and 193 channels. These structures appear to be located in front of the observed prominence, as they extend partially onto the solar disk and seem to absorb the background prominence emission. They are better visible in a movie that shows the temporal evolution in the SDO/AIA 193 and 304 channels which is available online.

The dark bubble that is well visible in the $\mathrm{H} \alpha$ line (Fig. 2) on the right side of the central absorption region can be spotted only in the SDO/AIA 193 channel. In the SDO/AIA 171 image, the bubble is covered up by the bright coronal emission (see also Parenti et al. 2012). Neither does the SDO/AIA 304 image reveal any sign of the bubble because the emission coming from its position is not distinguishable from the prominence elsewhere. This implies that the cool PCTR plasma is present along the LOS that traverses it. This plasma might lie behind, inside, or 
possibly in front of the actual bubble, as also suggested by the Hinode/SOT Ca II observations (see Sect. 2.2).

\subsection{SOHO/SUMER}

The SOHO/SUMER Lyman- $\alpha$ raster was obtained between $13: 43$ and 14:29 UT using the slit with dimensions of $0.278^{\prime \prime} \times$ $120^{\prime \prime}$. The first slit position of the raster was pointed at $X=510^{\prime \prime}$ with the lowest slit pixel at $Y=780^{\prime \prime}$. The scanning was made in the east-west direction and the dimensions of the obtained raster are $80^{\prime \prime} \times 120^{\prime \prime}$. Because of problems with the stepper motor moving the slit, scanning was not made at equidistant positions along the solar X coordinate. Moreover, the stepper motor often missed out a step, resulting in several subsequent observations made at the same slit position. Thus, although 180 observations were made, the slit was at only 115 different positions during the raster scanning. Correct slit positions and scanning-step distances were obtained directly from the stepper-motor encoder. At positions where multiple observations occurred we used the averaged values of the specific intensity at each pixel along the slit.

Three spectral windows from the whole SOHO/SUMER spectra displayed on detector $\mathrm{B}$ were recorded. The first

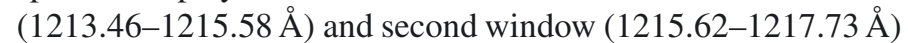
cover the Lyman- $\alpha$ line profile, while the third window (1237.87-1239.98 ^) contains the N V 1238.82 A line. The first and third observed spectral windows lie on the higher sensitivity $\mathrm{KBr}$ part of the detector, while the second window lies on the less sensitive bare part. This causes an increase of the noise in the red part of the Lyman- $\alpha$ line spectra (second window). Moreover, the boundary between $\mathrm{KBr}$ and the bare parts of the detector is not sharp or strictly vertical, which causes an additional unreliability of the red part of the Lyman- $\alpha$ profile calibration. Data were calibrated using SolarSoft procedures for the SOHO/SUMER data reduction and the SOHO/SUMER Data Cookbook (Schühle 2003). The data reduction is also explained in detail by Carlsson et al. (1997) and the wavelength calibration by Hansteen et al. (2000). The absolute intensity calibration of these SOHO/SUMER Lyman- $\alpha$ data is further complicated by observational technique, which requires a partial closure of the telescope aperture. This results in a decrease of the number of counts on the detector that needs to be increased by a factor of $5 \pm 1 \%$ to obtain the absolute intensity calibration (Curdt 2013, priv. comm.).

The raster image of the Lyman- $\alpha$ integrated intensities is shown in Fig. 4, top. We used the raster of the integrated intensity of the N V $1238.82 \AA$ line (Fig. 4, bottom) to determine the position of the solar limb (black dashed line). We note that the prominence is nearly invisible in the N V $1238.82 \AA$ line except for the very weak central column. This could be attributed to the N v $1238.82 \AA$ line formation temperature (its contribution function peaks at $\log T_{\max }[\mathrm{K}]=5.3$ ), which coincides with the local minimum of the prominence differential emission measure (DEM) curves derived from observations (see Wiik et al. 1993; Cirigliano et al. 2004; Parenti \& Vial 2007; Gunár et al. 2011b). The plane-of-the-sky extension of the prominence visible in the Lyman- $\alpha$ line is similar to the SDO/AIA 304 images (Fig. 3), although the hydrogen Lyman- $\alpha$ emission is associated with the cool prominence plasma with temperatures below $10000 \mathrm{~K}$. We can also resolve some of the dark elongated structures visible in the SDO/AIA 304 channel, but we are not able to identify the bubble. These similarities are due to the extremely high optical thickness of the Lyman- $\alpha$ line that reaches values of the order
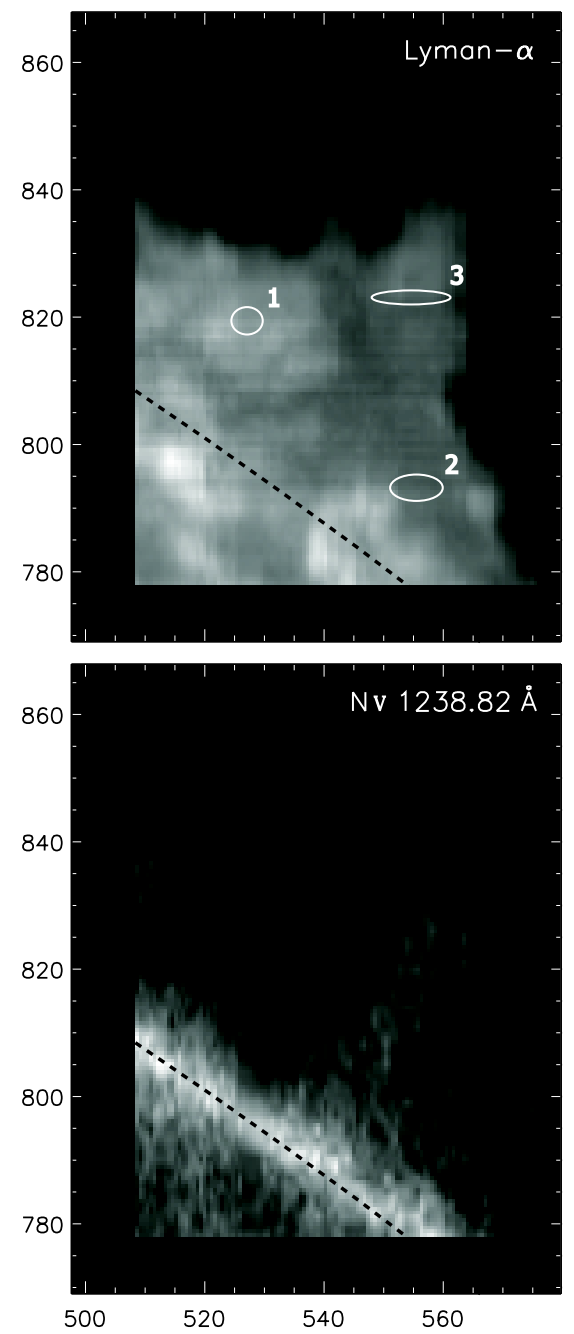

Fig. 4. SOHO/SUMER Lyman- $\alpha$ and N v $1238.82 \AA$ integrated intensity raster images obtained between 13:43 and 14:29 UT. Axes give the solar $X$ and $Y$ coordinates in arcsec. White ellipses indicate areas 1,2, and 3 .

of $10^{3}-10^{5}$ or even more in the line centre (see Gunár et al. 2008 and Fig. A.5 therein). Therefore the highest contribution to the observed Lyman- $\alpha$ intensity originates in a narrow PCTR region in front of the cool prominence fine-structure plasma. The temperature in this region can be as high as $50000 \mathrm{~K}$ (see analysis of the contribution functions in Heinzel et al. 2005). Discrepancies can be attributed to the fact that the SOHO/SUMER Lyman- $\alpha$ raster has a lower spatial resolution (below $1^{\prime \prime}$ ) and its scanning took 46 min during which the prominence evolved.

\subsection{Hinode/XRT}

We used two Hinode/XRT observations, one obtained at 15:37:45 UT using the Al-mesh filter and another obtained at 15:37:58 UT with the Ti-poly filter. Both have a field of view of $788^{\prime \prime} \times 788^{\prime \prime}$, with the lower left corner pointed at $X=92^{\prime \prime}$ and $Y=352^{\prime \prime}$. Data were binned by a factor of two immediately after the observation to conserve the Hinode telemetry bandwidth, resulting in a spatial resolution of 4 ". The response function of the Al-mesh filter has two maxima, corresponding to temperatures of $\log T[\mathrm{~K}] \approx 6$ and $\log T[\mathrm{~K}] \approx 6.9$ (see Fig. 7 of Golub et al. 2007). To be able to compare these data with the SDO/AIA 193 channel that observes plasma with a temperature 


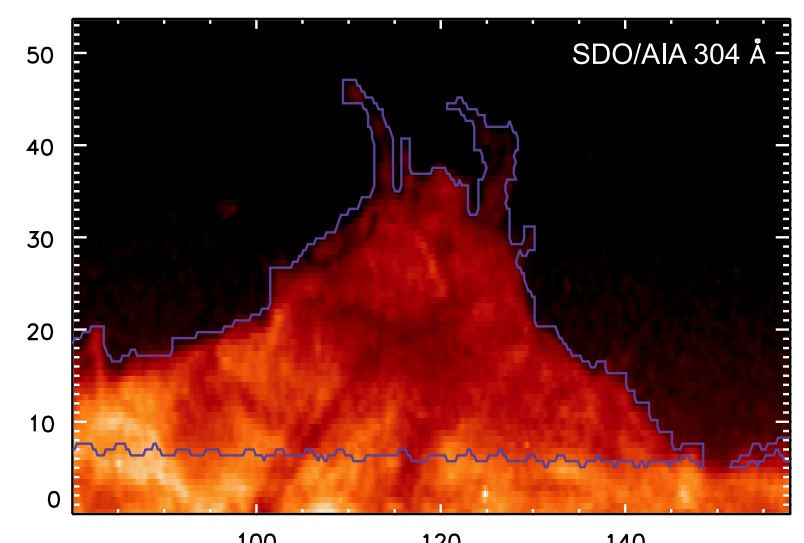

100

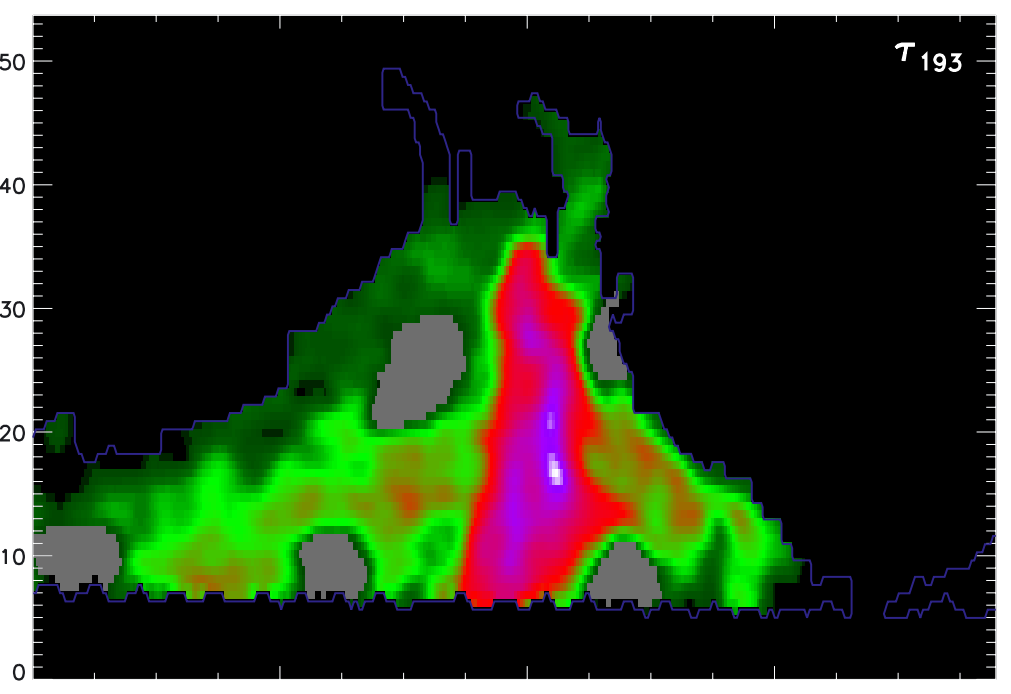

100

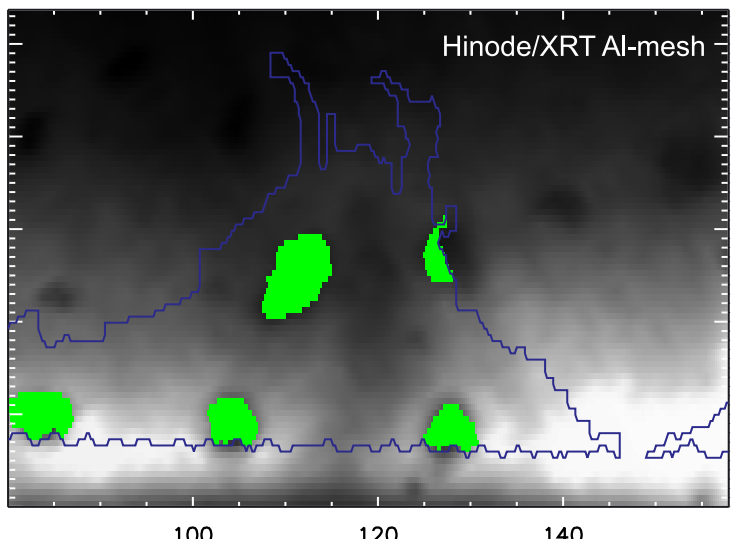

120

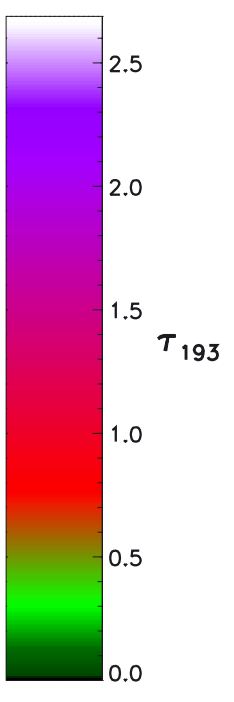

Fig. 5. Map of the optical thickness $\tau_{193}$ for the whole prominence, bottom, in comparison with the SDO/AIA 304 channel and Hinode/XRT Almesh observations. Note that the observed data were rotated to obtain the limb in the horizontal position. The $x$ - and $y$-axis give the dimensions in $1000 \mathrm{~km}$ and their origin was chosen arbitrarily. Green patches in the Hinode/XRT Al-mesh image and grey patches in the $\tau_{193}$ map cover pixels with corrupted or missing data.

of $\log T[\mathrm{~K}] \approx 6$, we had to remove the high-temperature contribution in Al-mesh data. This was made by using the Ti-poly filter with the sensitivity maximum at $\log T[\mathrm{~K}] \approx 6.9$. The sensitivity of the Ti-poly filter at $\log T[\mathrm{~K}] \approx 6$ is 20 times lower than Al-mesh filter, while at $\log T[\mathrm{~K}] \approx 6.9$ it is only twice as low (Golub et al. 2007). For the co-alignment of the Hinode/XRT images with the SDO/AIA observations we used the Al-mesh filter observations and the SDO/AIA 335 channel because the positions, shapes, and sizes of bright and dark structures on the disk in observations from both instruments correspond well. This can be explained by the fact that the thermal sensitivity of both the Hinode/XRT Al-mesh filter and the SDO/AIA 335 channel overlap in a wide temperature interval $(\log T[\mathrm{~K}]$ between 5.5 and 6.9). In Fig. 5 we plot a section of the full Hinode/XRT Almesh field of view with a size of $170^{\prime \prime} \times 210^{\prime \prime}$ that shows the cavity surrounding the prominence, which is faintly visible as a dark structure resembling that visible in the SDO/AIA $193 \mathrm{im}-$ age (Fig. 3). Here, green patches cover pixels with corrupted or missing data.

\section{Analysis of the optical thickness at $\mathrm{H} \alpha$ line centre}

The optical thickness of the $\mathrm{H} \alpha$ line centre $\tau_{0}(\mathrm{H} \alpha)$ represents an important constraint for a reliable prominence plasma diagnostics. In the absence of the $\mathrm{H} \alpha$ spectral observations (Hinode/SOT observed this prominence only in the $\mathrm{H} \alpha$ line wings) we can estimate $\tau_{\mathrm{o}}(\mathrm{H} \alpha)$ from the optical thickness of the SDO/AIA 193 channel observations. Anzer \& Heinzel (2005) showed that the optical thickness of hydrogen and helium resonance continua at $195 \AA$ is comparable to the optical thickness of the $\mathrm{H} \alpha$ line centre; the same is true at the $193 \AA$ wavelength. To calculate the optical thickness at this wavelength $\left(\tau_{193}\right)$ we used the method proposed by Heinzel et al. (2008), which is based on the fact that both the absorption and the blocking of the coronal emissivity contribute to the decrease of the coronal EUV intensity at $193 \AA$ while in the X-rays the absorption by a prominence hydrogen and helium plasma is negligible (Anzer et al. 2007). In the cavity surrounding the prominence, coronal intensities of both the EUV $193 \AA$ and the X-ray radiation are suppressed by the emissivity blocking (Heinzel et al. 2008). The intensities detected along a tangential cut through the prominence (and surrounding cavity) studied by Heinzel et al. (2008) show a decrease at the prominence position - shallower in the $\mathrm{X}$-rays because of the emissivity blocking alone and deeper in the coronal EUV radiation (in the TRACE $195 \AA$ ) because of the combination of the absorption and the emissivity blocking. However, we can assume that the coronal EUV and X-ray radiation in the cavity (outside of the prominence) is the same up to 
a multiplicative factor when we subtract from the $\mathrm{X}$-ray radiation the high-temperature contribution at $\log T[\mathrm{~K}] \approx 6.9$ (using the Hinode/XRT Ti-poly data, see Sect. 2.5). This leaves only the emission by plasma with a temperature of $\log T[\mathrm{~K}] \approx 6$. Thus we can calculate the $\tau_{193}$ with the following formula from Heinzel et al. (2008; see also Mein et al. 2001 and Schmieder et al. 2004)

$\tau_{193}=-\ln \left(1-\frac{1-r^{\prime}}{\alpha}\right)$.

Here $r^{\prime}$ is the ratio between the SDO/AIA $193 \AA$ intensity and the Hinode/XRT X-ray intensity multiplied by a factor to fit the EUV intensity in the cavity and corona and $\alpha$ is the factor of asymmetry of the coronal emissivity along the LOS. The X-ray intensity $I_{\mathrm{b}}(\mathrm{XRT})$ emitted along the LOS behind the prominence can be calculated as

$I_{\mathrm{b}}(\mathrm{XRT})=\alpha I(\mathrm{XRT})$,

where $I(\mathrm{XRT})$ is the X-ray intensity from the whole LOS at a prominence position detected by the Hinode/XRT. Then the X-ray intensity emitted from the corona in front of the prominence $I_{\mathrm{f}}(\mathrm{XRT})$ can be calculated as

$I_{\mathrm{f}}(\mathrm{XRT})=(1-\alpha) I(\mathrm{XRT})$.

In the case with more coronal emissivity behind the prominence than in front of it $\alpha$ is larger than 0.5. For the June 22, 2010 prominence we have estimated $\alpha$ to be around 0.5 from the comparison of intensities detected by the Hinode/XRT at the disk close to the limb and just above the limb close to the prominence position. This implies a symmetrical distribution of the coronal emissivity. Therefore we used in this study $\alpha=0.5$. By using Eq. (2) at each position in the prominence, a map of the optical thickness $\tau_{193}$ for the whole prominence can be constructed (Fig. 5, bottom). This map shows the same morphological features as the observations - central column with larger optical thickness surrounded on both sides by extended regions with lower optical thickness. Unfortunately, a large portion of the small bubble coincides with a patch of corrupted or missing data, which complicates its interpretation. However, a portion of this bubble located immediately to the right from this patch is unobscured and shows very low optical thickness of around 0.1.

\section{Magnetic field model}

As noted already in Sect. 2, the observed prominence has three well-defined morphological structures. The first is the central vertical region seen in absorption in SDO/AIA 171 and 193 channels and as the region of brightest emission in the Hinode/SOT Ca II H and $\mathrm{H} \alpha$ observations. Second is the extended region of numerous quasi-vertical fine-structure threads, very bright in the SOHO/SUMER Lyman- $\alpha$ raster image, located to the left from the central absorption region. The third region is characterized by the bubble lying underneath an area of tangled fine structures, that exhibits significant dynamics (see Berger et al. 2011). Any realistic magnetic field model must match all of these prominence features. To do that we used the model of Dudík et al. (2012) for the prominence magnetic field configuration.

The model of Dudík et al. (2012) is a linear force-free model that uses the method of Aulanier \& Demoulin (1998) and Aulanier et al. (1998). This type of modelling allows for matching of the general appearance of observed filaments and prominences with the visualized distributions of magnetic dips provided by the model. This technique allows us to predict the shape of $\mathrm{H} \alpha$ filaments (Aulanier et al. 2000) and when viewed from the side, the distribution of magnetic dips in these models appears as a prominence (Mackay \& van Ballegooijen 2009; Su $\&$ van Ballegooijen 2012). In these models, the filament channel is represented by stripes of opposite magnetic polarities located on both sides of the polarity inversion line. Parasitic bipoles perturb the inversion line and are associated with the formation of the filament feet of a chirality given by the sign of the electric current within the model (Aulanier \& Demoulin 1998; Aulanier et al. 1998). For more details on the prominence magnetic field modelling see reviews of Mackay et al. (2010), van Ballegooijen \& Su (2014), or Gunár (2014).

Dudík et al. (2012) adapted this type of modelling for quiescent polar-crown prominences with a characteristic cusp shape. These prominences were found to be a result of a strong, negatively sheared parasitic bipole present near the polarity inversion line within the filament channel. The resulting prominences were found to be feet-dominated, disconnected from the rest of the dips of the flux rope elsewhere in the model box, and exhibited large bubble underneath. These prominence magnetic field models with bubbles were found to provide a good approximation to the observed cusp-shaped polar crown prominences.

A generic model with unsheared bipole was also presented by Dudík et al. (2012), but no attempt was made there to compare it to the observations. This unsheared-bipole model is distinguished from the negatively sheared bipole one, since the distribution of prominence dips is not disconnected from that of the magnetic flux rope. The distribution of magnetic dips (downward-curved portions of the magnetic field lines) is visualized in Fig. 6 by the red colour-bars filling the dips up to one characteristic chromospheric pressure scale-height of $300 \mathrm{~km}$. Details of this visualization technique are given for example in Sect. 2 of Gunár et al. (2013). The distribution of dips is only interrupted by the large bubble that arises as a result of the bipole and its arcade-like field lines (pink field line in Fig. 6, top left). The bipole also creates two large feet on both its sides (see Dudík et al. 2012). The feet are also associated with a pair of magnetic null-points, whose spine and fan field lines are shown in yellow and blue in Fig. 6, top left.

This generic model with the unsheared bipole oriented in off-limb projection corresponding to that of the observed prominence (Fig. 6) reasonably matches the morphology of the June 22, 2010 prominence. The off-limb projection is determined as follows: first, the prominence is located at the north heliographic latitude of $\beta \approx 60^{\circ}$. Second, the prominence is observed off-limb, so that the local vertical must be inclined at an angle $\theta$ of nearly $90^{\circ}$ with respect to the line-of-sight (LOS). Third, the large bubble must be obscured by the prominence main body, that is, the angle $\phi$ between the prominence main axis and LOS must be nearly zero. We determined that the model projected at $\beta=60^{\circ}, \theta \approx 85-88^{\circ}$, and $\phi \approx 0-5^{\circ}$ provide a reasonable approximation to the observed shape of the prominence. For the projection shown in Fig. 6, top middle we used $\theta=85^{\circ}$ and $\phi=5^{\circ}$.

The dominant feature of the model is the prominence body consisting of densely packed magnetic dips. When viewed in the off-limb projection (Fig. 6, top middle), the prominence body clearly represents the observed central column seen in bright emission in the $\mathrm{H} \alpha$ and $\mathrm{Ca}$ II $\mathrm{H}$ lines and in absorption in the SDO/AIA 171 and 193 channels. Both the excess emission and the strong absorption are then due to the large amount of the prominence plasma (large column mass) located along any LOS 

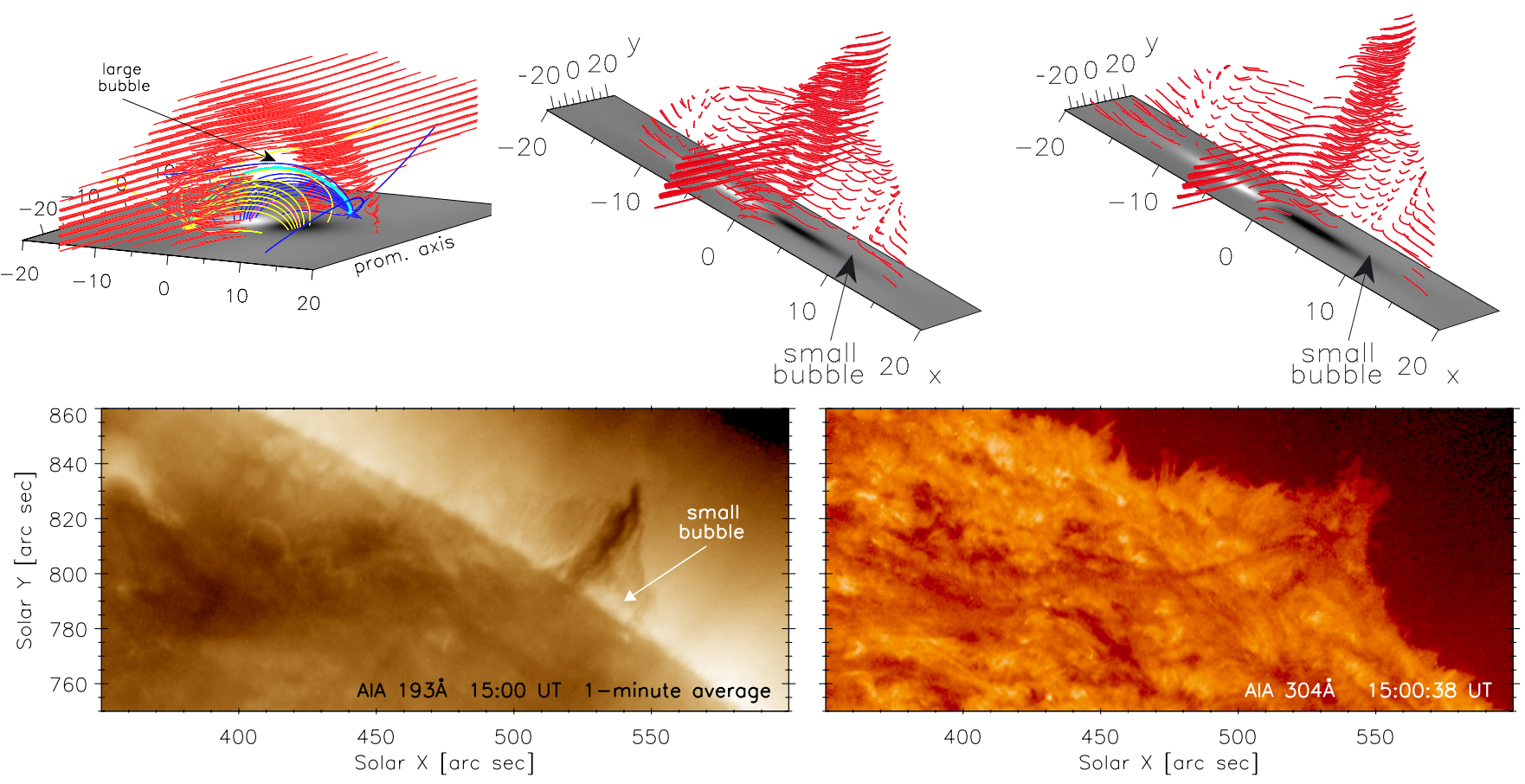

Fig. 6. Magnetic field model of the prominence compared to SDO/AIA observations. Top left: original magnetic field model of the prominence with symmetric unsheared bipole (Dudík et al. 2012, Fig. 6 therein). Magnetic dips up to a height of $300 \mathrm{~km}$ are shown in red. Spine and fan field lines of the positive and negative magnetic null-points are shown in yellow and blue. Arrow points to the large bubble within the prominence created by the bipole. Top middle: the same model, projected off-limb at $30^{\circ}$ of solar latitude. The small bubble within the right foot is denoted by the arrow. Top right: asymmetric model in the same off-limb projection. Dimensions are given in units of 1000 km. Bottom row: SDO/AIA 304 channel observations of the prominence together with the on-disk filament (right), while a weak filament channel is visible in SDO/AIA 193 channel $(l e f t)$.

traversing the prominence body. The prominence feet located on the two sides of the prominence body represent both the extended region of quasi-vertical fine structures and the region above the small bubble reported by Berger et al. (2011). We note here that the small bubble seems to correspond to a small gap in the right-hand prominence foot around the negative magnetic null-point (arrow in Fig. 6, top middle). We caution, however, that the magnetic field model employs a Cartesian geometry, so that the comparison with an off-limb prominence on a spherical Sun is only qualitative.

Nevertheless, a closer match to the observed shape of the prominence can be achieved by introducing an asymmetry. To do so, we moved the positive-polarity of the bipole twice as far away $(5000 \mathrm{~km})$ from the inversion line. This produces a curved central column similar to the observations in SDO/AIA 193 channel and a more extended left-hand foot. It also increases the size of the small bubble in the right-hand foot (Fig. 6, top right). In this scenario, a $\lambda$-like shape is recreated by an extension of the on-disk filament together with the central column (see Fig. 6, bottom right). Note that the observations of the on-disk filament in the SDO/AIA 304 channel (Fig. 6, bottom right) and the associated weak EUV filament channel in the 193 channel (Fig. 6, bottom left) support this scenario. We also note that a wide range of asymmetric models can be produced by altering the bipole position and magnetic flux. However, the photospheric magnetic field at the limb is unconstrained by observations and therefore we did not explore the possible configurations any further. Finally, it is important to note that the local magnetic field directions in both the symmetric and asymmetric models presented in Fig. 6, top are very similar.

In the scenario described by the models, the feet regions on both sides of the central column would have significantly lower column mass than the central column. This is due to the much smaller number of magnetic dips located along any LOS traversing them. This is consistent with our analysis of the $\mathrm{H} \alpha$ line centre optical thickness (Sect. 3), which shows significantly larger optical thickness in the central column (main prominence body) than on its sides (see Fig. 5). We note, however, that it is not the aim of this model to exactly recreate the precise configuration of the June 22, 2010 prominence. Instead, the model provides a reasonable estimate of the magnetic field geometry in different parts of the prominence.

\section{Analysis of the Lyman- $\alpha$ data}

To analyse the observed Lyman- $\alpha$ data in detail, we selected three distinct parts of the Lyman- $\alpha$ raster that correspond to three main morphological features of the June 22, 2010 prominence described in Sect. 2.2, see also the above section for details of their magnetic structure. AREA1 lies in the region of the brightest Lyman- $\alpha$ emission corresponding to the location of numerous quasi-vertical fine-structure threads. AREA2 is located in the dynamical region just above the bubble. AREA3 lies in the uppermost part of the central vertical region. These areas are indicated in Figs. 2-4.

\subsection{AREA1 - prominence fine structures}

AREA1 lies in a rather typical quiescent prominence region with many quasi-vertical fine structures (see Fig. 2). To analyse it in detail we selected a small cluster of clearly reversed profiles that are only mildly affected by noise. In Fig. A.1 we show a small portion of the Lyman- $\alpha$ profiles from AREA1 where the profiles we selected for the analysis are marked in red. 
We compared these observed profiles with the synthetic Lyman- $\alpha$ spectra obtained by the 2D prominence fine-structure models developed by Heinzel \& Anzer (2001). These depict the fine structures of prominences as vertically infinite 2D threads embedded in a horizontal magnetic field. The variation of all quantities takes place only in the horizontal plane parallel to the solar surface, while everything is uniform in the vertical direction (see sketch in Fig. 7a). These threads are in the local 2D magnetohydrostatic equilibrium of the Kippenhahn-Schlüter type (Heinzel \& Anzer 2001) and their temperature structure is specified empirically to encompass both the central cool part and two different forms of the PCTR, narrow layer with a steep temperature gradient in the direction perpendicular to the magnetic field and more extended region with gradual rise of the temperature oriented along the field lines. To determine the synthetic hydrogen spectra emerging from these prominence fine-structure threads, we solved 2D multi-level non-LTE radiative transfer. We adopted the partial frequency redistribution (PRD, Heinzel et al. 1987; Paletou 1995) for the Lyman- $\alpha$ and Lyman- $\beta$ lines. The details of the method and models are given in Heinzel \& Anzer (2001) and Heinzel et al. (2005; see also the review by Gunár 2014). Here we did not employ the multi-thread models with stochastic LOS velocity field (developed by Gunár et al. 2008) because without other Lyman line observations than the Lyman- $\alpha$ and because we lack $\mathrm{H} \alpha$ spectra we cannot determine the number of threads of these models. Moreover, because of the very high optical thickness of the Lyman- $\alpha$ line in the centre and near wings, its emission is formed mostly in the foremost PCTR layer. Therefore, we did not attempt here to find a prominence fine-structure model that produces synthetic spectra that agree in detail with observations. Instead, we used a single-thread configuration of a model that was previously used for analysis of a similar prominence observations - MoDEL3_6000 (input parameters are listed in Table 1). This model was used by Gunár et al. (2012) to study the polar-crown prominence observed on April 26, 2007, but it also qualitatively agrees with the Lyman- $\alpha$ profiles of the prominence studied here. In Fig. 8 we show the comparison of the selected observed profiles (marked by black lines with error-bars) and the synthetic Lyman- $\alpha$ profiles produced by MODEL3_6000 (red lines). We plot the synthetic profiles at 83 positions along the length of the thread with the LOS perpendicular to the magnetic field lines (for more details see Heinzel et al. 2005). This model agrees reasonably well with the observed Lyman- $\alpha$ spectra from AREA1 and thus shows that this part of the June 22, 2010 prominence has indeed properties of a typical quiescent prominence. Unfortunately, we cannot directly compare this prominence with that observed on April 26, 2007 (Gunár et al. 2012) because for the June 22, 100 prominence we only have the Lyman- $\alpha$ observations, while for the April 26, 2007 prominence there are no Lyman- $\alpha$ observations but the higher Lyman lines are available.

\subsection{AREA2 - above the bubble}

The Lyman- $\alpha$ profiles from AREA2 that lie above the bubble have significantly lower intensities than the Lyman- $\alpha$ spectra from the other two studied locations (see Fig. A.2). However, the available SOHO/SUMER raster observations took $46 \mathrm{~min}$ to complete and thus cannot adequately account for the dynamical character of the area adjacent to the bubble that typically exhibits variations on the scale of several minutes (Berger et al. 2011). Accordingly, we can only speculate about the possible explanations of the low observed Lyman- $\alpha$ intensities in AREA2. One of them might be the decrease of the plane-of-the-sky filling

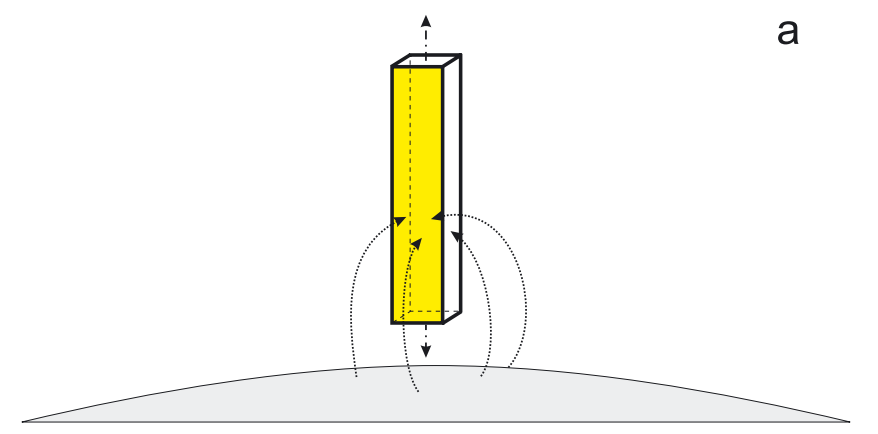

b

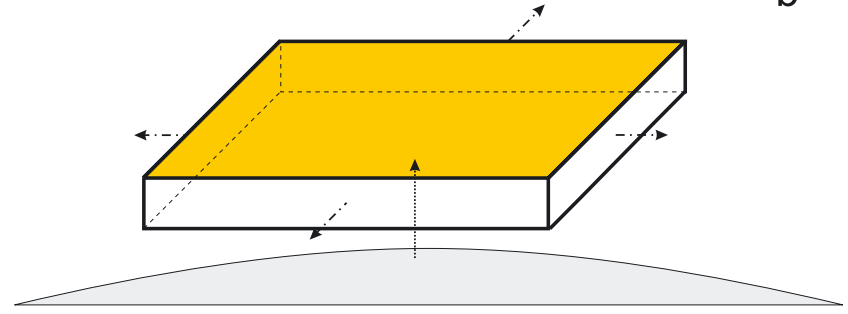

C

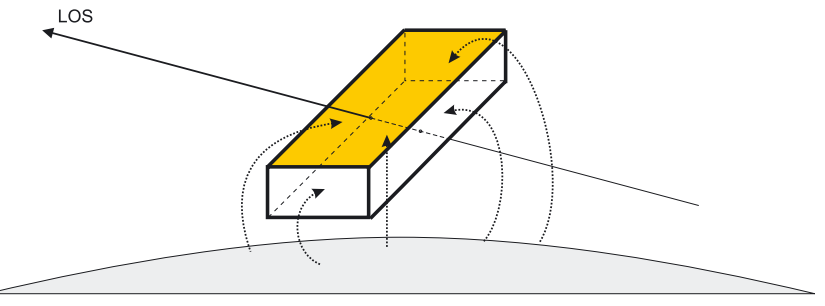

Fig. 7. Sketch of the vertically infinite 2D prominence fine structure model used for analysis of AREA1 a), 1D horizontal-slab filament model b), and a general 3D model c). Dotted arrows indicate the incident radiation from the solar surface, while the dash-dotted lines show the directions of the infinite dimensions. Colour highlights the observed surfaces. An LOS drawn in panel c) shows the representative direction towards the observer for AREA3.

factor due to the passage of plumes (small-scale dark areas visible in the $\mathrm{H} \alpha$ and $\mathrm{Ca}$ II H Hinode/SOT observations) with dimensions below the spatial and/or temporal resolution of the SOHO/SUMER (Berger et al. 2010, 2011). This would suggest that the Lyman- $\alpha$ radiation coming from the position of the passing plumes is lower than that from their surroundings. However, the apparent invisibility of the dark bubble in the Lyman- $\alpha$ raster (and also in the SDO/AIA 304 observations) suggests that the effect of the much smaller plumes on the total Lyman- $\alpha$ intensity might be negligible. Another option could be an overall decrease of the intensity from the whole AREA 2 caused, for example by fewer magnetic dips filled with prominence plasma because of the dynamical character of this region. Or it might be caused by the diminished number of the magnetic dips themselves, as suggested by the prominence magnetic field simulations presented in Sect. 4. Because of this ambiguity, we did not attempt to analyse this area in greater detail. We note that the dynamics of the plumes rising through the prominence fine structures were also studied by Hillier et al. (2012a). 

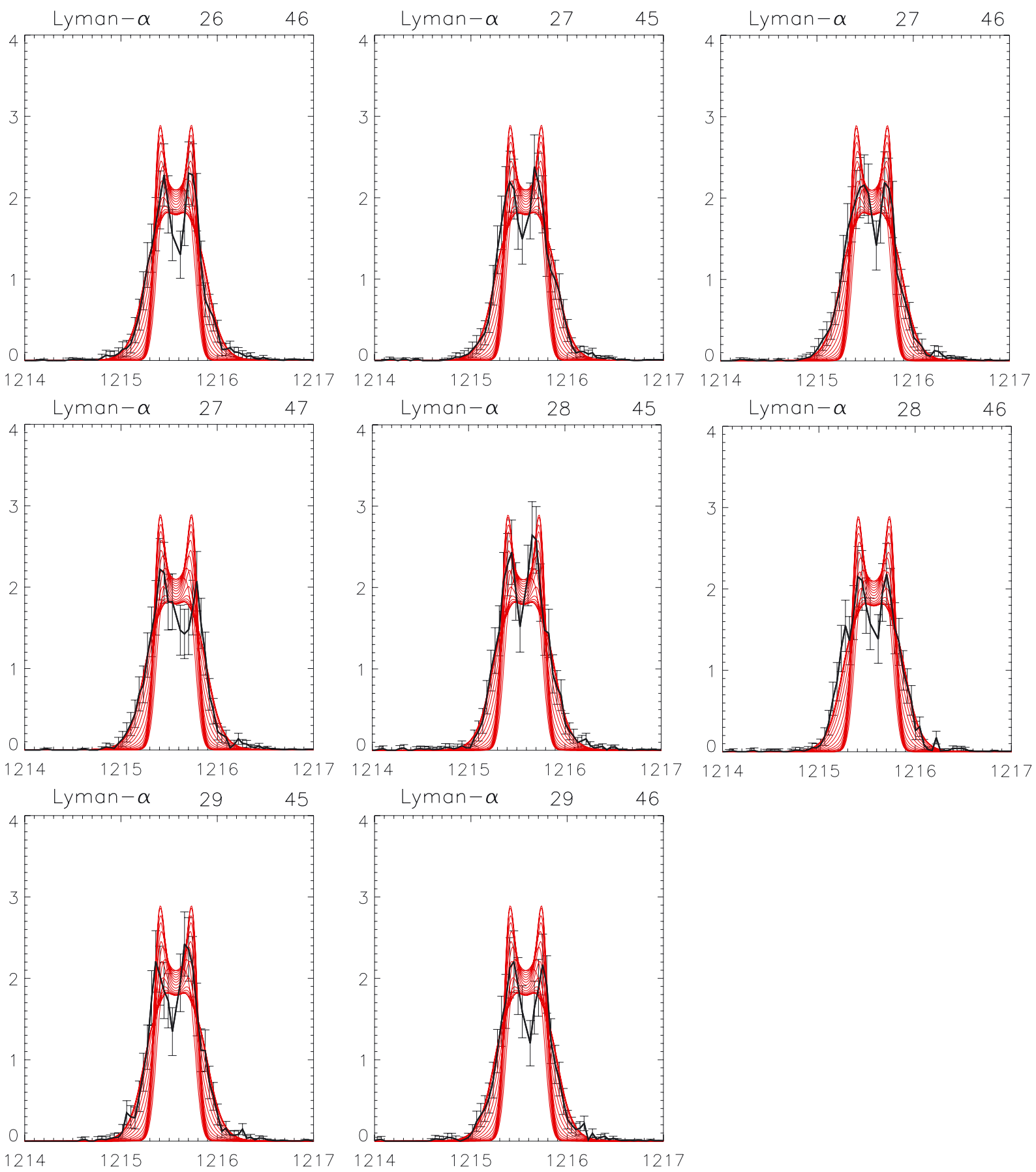

Fig. 8. Comparison of the selected observed profiles (black lines with error bars) and the synthetic Lyman- $\alpha$ profiles produced by MoDEL3_6000. We plot the synthetic profiles at 83 positions along the length of the thread. Numbers above each panel indicate the $x$ and $y$ positions in the SOHO/SUMER raster. The $x$-axis gives the wavelength in $\AA$, the $y$-axis the specific intensity in $10^{-8} \mathrm{erg} \mathrm{s}^{-1} \mathrm{~cm}^{-2} \mathrm{sr}^{-1} \mathrm{~Hz}^{-1}$.

\subsection{AREA3 - similar to a filament}

AREA3 is located at the top part of the vertical region of strong absorption seen in the SDO/AIA 171 and 193 channels (Fig. 3). The Lyman- $\alpha$ profiles from this region have specific intensities significantly lower than those from AREA1 (see Fig. A.3), but higher than those from AREA2. The Lyman- $\alpha$ central intensities of these profiles are below $1 \times 10^{-8} \mathrm{erg} \mathrm{s}^{-1} \mathrm{~cm}^{-2} \mathrm{sr}^{-1} \mathrm{~Hz}^{-1}$ and thus well below the theoretical limit for the prominence Lyman- $\alpha$ central intensities. This limit is approximately $1.3 \times$ $10^{-8} \mathrm{erg} \mathrm{s}^{-1} \mathrm{~cm}^{-2} \mathrm{sr}^{-1} \mathrm{~Hz}^{-1}$ for the synthetic Lyman- $\alpha$ central intensity produced by the isothermal isobaric $1 \mathrm{D}$ prominence models at a height of $10000 \mathrm{~km}$ (Heinzel et al. 1987) and is even higher for models including the PCTR. These values correspond to a minimum amount of the Lyman- $\alpha$ line-centre radiation emerging from a prominence surface that is directly illuminated from the solar surface, taking into account an appropriate dilution factor. The lower observed Lyman- $\alpha$ central intensities from the AREA3 suggest that we are observing part of the prominence that is not directly illuminated from the solar surface.

This idea is supported by the morphology of this prominence (see Sect. 4), which also suggests that at the position of AREA3 we see the upper part of the prominence spine from the top with an angle that is more akin to observations of filaments. The 
upper surface of filaments (see Fig. 7b) is not irradiated from the solar surface (irradiation from the corona in hydrogen spectral lines is quite negligible) and therefore the limit for the emerging Lyman- $\alpha$ central intensity is much lower than that for prominences. The emerging radiation in case of a filament (observed surface is not directly illuminated) is determined by the source function, which is driven mainly by the diffusion of radiation scattered from all irradiated surfaces. In contrast, a large portion of the radiation emerging from a directly illuminated surface of a prominence is formed by a scattering of the incident radiation into the direction towards the observed.

Interestingly, synthetic Lyman- $\alpha$ profiles produced by $1 \mathrm{D}$ models approximating entire filament by a horizontal 1D plane-parallel slab infinite in both horizontal directions and finite in the vertical direction (see Fig. 7b), such as those of Schwartz et al. (2012), are similar to the observed spectra from AREA3. However, such 1D filament models cannot be realistically used to explain the observed Lyman- $\alpha$ profiles from AREA3, because they assume the incident radiation from the solar surface that irradiates the bottom surface of the filament slab to be explicitly in the LOS direction. Clearly, that is not the case for AREA3, which is observed above the solar limb, and thus any LOS towards the observer does not intersect the solar surface. Therefore, to obtain realistic synthetic spectra comparable to those of AREA3, we would have to assume a general 3D model with an LOS intersecting the top (not irradiated surface) but not the solar surface (see sketch in Fig. 7c). Emerging radiation from such a top surface without a direct illumination from the solar surface would strongly depend on the scattering of the incident radiation at the side surfaces. However, such a model is beyond the scope of this work.

\section{Discussion}

\subsection{Prominence-corona transition region}

It is generally assumed that most of the dense cool plasma of the prominence fine structures lies in dips of the prominence magnetic field. These dips can be either strongly affected or indeed be caused by the weight of the prominence fine-structure plasma, or they can occur as the result of, for example, forcefree nature of the magnetic field configurations (see reviews of Mackay et al. 2010 and Gunár 2014). In case of dips, the temperature variation of the PCTR plasma is governed by two distinct regimes. In the direction across the field lines, the temperature steeply rises from the central cool parts towards the coronal values. This is caused by inhibited thermal conductivity across the magnetic field lines. On the other hand, the thermal conductivity is efficient along the field lines transporting the heat from the corona into the prominence, resulting in a much gentler temperature gradient in the direction parallel to the field lines. This, in combination with a larger extension of individual plasma structures in the direction along the field, results in a rather extended PCTR located along the dipped field lines spreading out from the cool central parts. On the other hand, the PCTR layer that envelopes the central cool parts in the direction perpendicular to the magnetic field is very narrow and exhibits a steep temperature gradient. This scenario suggests that most of the PCTR material might be located in the extended PCTR fine structures that are connected to the cool plasma lying at the bottom of the dipped field lines. Such structures were shown by Karpen et al. (2006) and Luna et al. (2012) to form naturally, in 1D geometry, by applying heating at the foot-points of the dipped field lines. This causes chromospheric plasma evaporation, its transportation along the field lines, and subsequent cooling (due to the radiative losses) and condensation, thus forming the smallscale cool plasma structures. If these are formed inside the magnetic dips, they remain in the corona for long time periods. The temperature in these $1 \mathrm{D}$ models decreases gradually along the field lines from the coronal to the prominence values. We note that these prominence formation models do not consider temperatures below $30000 \mathrm{~K}$ because at lower temperatures detailed radiative-transfer treatment of radiative losses is necessary.

The 2D prominence fine structure models of Heinzel \& Anzer (2001) used in Sect. 5.1 also accommodate a similar temperature structure that includes two distinct PCTR forms, a narrow one with a steep temperature gradient perpendicular to the magnetic field, and a much more extended one with a gradual rise of the temperature along the magnetic field. This means that in these models most of the PCTR plasma is located in the extended regions that protrude in the direction of the field lines on both sides of the cool prominence cores. If observed on the solar disk as a filament, these PCTR fine structures would in aggregate form the EUV extensions surrounding the $\mathrm{H} \alpha$ filaments, as considered for instance by Schwartz et al. (2006).

The 2D prominence fine structure models of Heinzel \& Anzer (2001) were shown to produce the synthetic hydrogen spectra in a very good agreement with the multiple prominence observations (see e.g. Gunár et al. 2010). Moreover, comparison of the synthetic DEM curves derived from the temperature and electron density structure produced by these models also agrees well with some of the observed DEM curves (Gunár et al. 2011a,b). This suggests that the plasma structure of these models is not far from the real prominence plasma distribution. We note that these 2D models do not consider temperatures above $100000 \mathrm{~K}$ where hydrogen is fully ionized. The prominence plasma temperature variation in the direction parallel to the magnetic field that reaches significantly higher values (up to $450000 \mathrm{~K}$ ) was produced by Anzer \& Heinzel (2008), who studied the prominence energy balance. This work also showed that along the magnetic field lines the temperature decreases over a large geometrical distance, resulting in a considerably extended PCTR.

The 3D prominence magnetic field simulations presented in Sect. 4 allowed us to produce the whole-prominence configurations of the magnetic field with distributions of dips that qualitatively corresponded to the studied prominence. If we were to fill these dips with realistic prominence plasma (e.g. by the method of Gunár et al. 2013), we would produce a prominence model with a large proportion of the PCTR plasma located in the PCTR fine structures that lie inside the magnetic dips at both sides of the cool prominence material. These individual PCTR fine structures would together appear as an extended PCTR halo that surrounds the cool prominence parts. However, the question remains whether all dips present in the prominence magnetic field configurations host the cool prominence plasma, or what kind of material fills the dips without the cool prominence cores, plasma under coronal conditions, or a PCTR plasma that does not reach the low prominence temperatures. This depends on the energy balance within the dips, a question that has so far not been studied in detail.

\subsection{Bubble below the prominence}

Dark bubbles appearing below the quiescent prominence bodies are now a well-documented fact, see for example Berger et al. (2011) and the review by Berger (2014). However, their nature, origin, and the mechanisms of their evolution, together 
with the apparent production of plumes are still not understood. Our analysis of the multi-instrument observations of the June 22, 2010 prominence allows us to illuminate some of these open questions. We note that the following discussion only considers the small bubble visible in the lower right corner, see e.g. Figs. 2 and 6 , bottom left.

As we describe in Sect. 2, observations of this prominence suggest that at least some material at cool PCTR temperatures (up to $50000 \mathrm{~K}$ ) is located along the LOS traversing the bubble. This is evidenced by the invisibility of the bubble in the SOHO/SUMER Lyman- $\alpha$ (Fig. 4) and in the SDO/AIA 304 observations (Fig. 3), that is, in lines with high optical thickness. Moreover, Hinode/SOT observations in the CaII line (Fig. 2) show the bubble partially obscured, which also implies the occurrence of cool prominence material at about $10000 \mathrm{~K}$. The column density of this cool prominence plasma is rather low, which is supported by the low values of the $\tau_{193}$ (equivalent to the optical thickness at the $\mathrm{H} \alpha$ line centre) that are below 0.2 (see Fig. 5). However, even the broad set of multi-instrument observations analysed in this work is unable to provide a conclusive answer to the question where this emission is located: whether it is inside, in front of, or behind the actual bubble.

This issue can be illuminated by the 3D prominence magnetic field simulations of Dudík et al. (2012), which accommodate a large region devoid of the magnetic dips that represent the observed bubble in a correct projection (see Sect. 4). This model shows that the bubble is mainly surrounded by a layer containing few magnetic dips (as compared to the main prominence body) that might accommodate plasma that emits the radiation observed at the position of the bubble. This would suggest that this emission originates in front of or behind the bubble and that the actual bubble is filled with rarified, perhaps coronal cavity plasma. Such a bubble would be no obstacle to the background coronal radiation and would represent an open window through the prominence body. The excess emission seen inside bubbles in the SDO/AIA 171 observations (reported by Berger et al. 2011) might be explained by the PCTR emission originating in the few magnetic dips that surround the bubble and might also accommodate the cool prominence material.

We note that arguments that bubbles in polar-crown prominences are open windows were previously presented by Dudík et al. (2012), who studied the cusp-shaped polar-crown prominences and their bubbles. However, these bubbles were found to be associated with a pair of null-points with opposite signs, connected by a separator delineating the boundary between the bubble and the prominence body composed of magnetic dips. The model presented here (Sect. 4) is different, since the small bubble is an opening within only one of the feet, and is associated with only one null-point. The large bubble associated with both null-points is obscured in the projection corresponding to the prominence observed off-limb (Fig. 6). This suggests that a single null-point may be sufficient to produce an opening within the prominence foot that resembles the observed bubble.

Finally, we note that any analysis of the bubble is additionally complicated by its dynamical nature. The prominence magnetic field model presented here is quasi-static and cannot be directly compared with the dynamics of the observed bubble that produces small-scale plumes rising through the prominence body (Berger et al. 2011). However, the association of the small bubble with the null-point indicates that the reconnection at this null-point might lead to formation of dynamic features. The location of the null-point and the associated change of direction of the dipped magnetic field lines above it (Fig. 6) is consistent with the observed location of the plume (Berger et al. 2011, Fig. 1 therein).

\section{Conclusions}

This analysis of the June 22, 2010 prominence shows the importance of the coordinated multi-instrument observation campaigns and of combining them with the multi-disciplinary modelling.

One interesting aspect of this prominence is the fact that our 2D models of prominence fine structures are not able to reproduce the prominence spectra from the whole observed area, although they apparently work well for the part dominated by the quasi-vertical threads. We note that these models were developed to model such vertical fine-structure threads. On the other hand, in the most prominent feature of this prominence, the central column that represents the projection of the main prominence body, such models are unable to reproduce the observed reversed Lyman- $\alpha$ line profiles with low central intensities. This is true even after taking into account the effect of the LOS orientation with respect to the magnetic field studied for example by Schmieder et al. (2007). The observed central Lyman- $\alpha$ intensities are below the theoretical limit for prominence models with the observed surface directly illuminated from the solar surface (see discussion in Sect. 5.3). This, together with the morphology of studied prominences (see Sect. 4), suggests that at the upper portion of the central column we observe the top part of the prominence/filament spine that is not directly irradiated from the solar surface.

The multi-disciplinary study of this prominence also allowed us to shed some light on the nature of the prominence-corona transition region. By employing radiative transfer and magnetic field modelling, we analysed the multi-instrument observations, which allow us to argue that a large part of the PCTR plasma is located in the PCTR fine structures inside the magnetic dips at both sides of the cool prominence material that extends in the direction parallel to the magnetic field. An extended PCTR halo formed by the sum of the individual PCTR fine structures probably surrounds the cool prominence parts. However, it is not yet understood whether all prominence magnetic dips host the cool prominence plasma, or if they contain the plasma under coronal or PCTR conditions. We note that such dips filled with PCTR plasma, if existent, might substantially contribute to the overall amount of the PCTR plasma in prominences.

We also showed that the generic magnetic field model of Dudík et al. (2012) with unsheared bipole is able to reproduce the observed morphology of the prominence, if oriented in the correct off-limb projection (see Sect. 4). In this model, the filament flux-rope is perturbed by a parasitic bipole. This bipole creates a large bubble underneath the flux-rope and is also associated with a pair of null-points and two large prominence feet. When viewed off-limb, the central part of the filament above the bubble resembles the prominence vertical column observed in absorption in the SDO/AIA 193 channel, while the left-hand and right-hand parts of the observed prominence correspond to both modelled feet. Moreover, introducing an asymmetrical bipole can improve the qualitative match between the model and observations. Comparison of the observations with the model also showed that the small observed bubble corresponds to an opening within the modelled right-hand foot at the location of one of the magnetic null-points. In this respect, the small bubble is different from the large bubbles studied by Dudík et al. (2012), which are associated with both feet and both null-points. The observed bubble is well visible in some wavelengths, but 
completely unidentifiable in others. We argue that this is because the void is surrounded by relatively narrow layers of magnetic dips that form the prominence feet. In the off-limb projection presented here, this can account for both their visibility in optically thin lines (either cool prominence or hot PCTR lines) and their invisibility in optically thick lines formed at lower PCTR temperatures.

Acknowledgements. S.G. acknowledges support from the European Commission via the Marie Curie Actions - Intra-European Fellowships Project No. 328138. S.G., P.S., and P.H. acknowledge the support from grant P209/12/0906 of the Grant Agency of the Czech Republic. P.S. acknowledges support from the Slovak Research and Development Agency under the contract No. APVV-0816-11. J.D. acknowledges support from the Royal Society via the Newton Fellowships programme. J.J. acknowledges the support from grant P209/12/0287 of the Grant Agency of the Czech Republic. Work of S.G., P.S., P.H., and J.J. was supported by the project RVO: 67985815. Work of P.S. was supported by the project VEGA 2/0108/12 of the Science Grant Agency. P.S. acknowledges the support from Observatoire de Paris, Section de Meudon. B.S. thanks for the support from the Astronomical Institute of the Academy of Sciences of Czech Republic. Hinode is a Japanese mission developed and launched by ISAS/JAXA, with NAOJ as domestic partner and NASA and STFC (UK) as international partners. It is operated by these agencies in cooperation with ESA and NSC (Norway).

\section{References}

Andretta, V., Del Zanna, G., \& Jordan, S. D. 2003, A\&A, 400, 737

Anzer, U., \& Heinzel, P. 2005, ApJ, 622, 714

Anzer, U., \& Heinzel, P. 2008, A\&A, 480, 537

Anzer, U., Heinzel, P., \& Fárnik, F. 2007, Sol. Phys., 242, 43

Aulanier, G., \& Demoulin, P. 1998, A\&A, 329, 1125

Aulanier, G., Demoulin, P., van Driel-Gesztelyi, L., Mein, P., \& Deforest, C. 1998, A\&A, 335, 309

Aulanier, G., Srivastava, N., \& Martin, S. F. 2000, ApJ, 543, 447

Berger, T. 2014, in IAU Symp. 300, 15

Berger, T., Testa, P., Hillier, A., et al. 2011, Nature, 472, 197

Berger, T. E., Slater, G., Hurlburt, N., et al. 2010, ApJ, 716, 1288

Boerner, P., Edwards, C., Lemen, J., et al. 2012, Sol. Phys., 275, 41

Carlsson, M., Judge, P. G., \& Wilhelm, K. 1997, ApJ, 486, L63

Cirigliano, D., Vial, J., \& Rovira, M. 2004, Sol. Phys., 223, 95

Del Zanna, G., O’Dwyer, B., \& Mason, H. E. 2011, A\&A, 535, A46

Dudík, J., Aulanier, G., Schmieder, B., Bommier, V., \& Roudier, T. 2008, Sol. Phys., 248, 29

Dudík, J., Aulanier, G., Schmieder, B., Zapiór, M., \& Heinzel, P. 2012, ApJ, 761, 9

Golub, L., Deluca, E., Austin, G., et al. 2007, Sol. Phys., 243, 63

Goode, P. R., Denker, C. J., Didkovsky, L. I., Kuhn, J. R., \& Wang, H. 2003, J. Korean Astron. Soc., 36, 125

Gouttebroze, P., \& Heinzel, P. 2002, A\&A, 385, 273

Gouttebroze, P., Heinzel, P., \& Vial, J. C. 1993, A\&AS, 99, 513
Gunár, S. 2014, in IAU Symp., 300, 59

Gunár, S., Teriaca, L., Heinzel, P., \& Schühle, U. 2006, in SOHO-17, 10 Years of SOHO and Beyond, ESA SP, 617

Gunár, S., Heinzel, P., Schmieder, B., Schwartz, P., \& Anzer, U. 2007, A\&A, 472,929

Gunár, S., Heinzel, P., Anzer, U., \& Schmieder, B. 2008, A\&A, 490, 307

Gunár, S., Schwartz, P., Schmieder, B., Heinzel, P., \& Anzer, U. 2010, A\&A, 514, A43

Gunár, S., Heinzel, P., \& Anzer, U. 2011a, A\&A, 528, A47

Gunár, S., Parenti, S., Anzer, U., Heinzel, P., \& Vial, J.-C. 2011b, A\&A, 535, A122

Gunár, S., Mein, P., Schmieder, B., Heinzel, P., \& Mein, N. 2012, A\&A, 543, A93

Gunár, S., Mackay, D. H., Anzer, U., \& Heinzel, P. 2013, A\&A, 551, A3

Hansteen, V. H., Betta, R., \& Carlsson, M. 2000, A\&A, 360, 742

Heinzel, P. 2007, in The Physics of Chromospheric Plasmas, eds. P. Heinzel, I. Dorotovič, \& R. J. Rutten, ASP Conf. Ser., 368, 271

Heinzel, P., \& Anzer, U. 2001, A\&A, 375, 1082

Heinzel, P., Gouttebroze, P., \& Vial, J.-C. 1987, A\&A, 183, 351

Heinzel, P., Anzer, U., \& Gunár, S. 2005, A\&A, 442, 331

Heinzel, P., Schmieder, B., Fárník, F., et al. 2008, ApJ, 686, 1383

Hillier, A., Berger, T., Isobe, H., \& Shibata, K. 2012a, ApJ, 746, 120

Hillier, A., Isobe, H., Shibata, K., \& Berger, T. 2012b, ApJ, 756, 110

Karpen, J. T., Antiochos, S. K., \& Klimchuk, J. A. 2006, ApJ, 637, 531

Kosugi, T., Matsuzaki, K., Sakao, T., et al. 2007, Sol. Phys., 243, 3

Labrosse, N., \& Gouttebroze, P. 2001, A\&A, 380, 323

Labrosse, N., Heinzel, P., Vial, J., et al. 2010, Space Sci. Rev., 151, 243

Lemen, J. R., Title, A. M., Akin, D. J., et al. 2012, Sol. Phys., 275, 17

Luna, M., Karpen, J. T., \& DeVore, C. R. 2012, ApJ, 746, 30

Mackay, D. H., \& van Ballegooijen, A. A. 2009, Sol. Phys., 260, 321

Mackay, D. H., Karpen, J. T., Ballester, J. L., Schmieder, B., \& Aulanier, G. 2010, Space Sci. Rev., 151, 333

Mein, N., Schmieder, B., DeLuca, E. E., et al. 2001, ApJ, 556, 438

O’Dwyer, B., Del Zanna, G., Mason, H. E., Weber, M. A., \& Tripathi, D. 2010, A\&A, 521, A21

Paletou, F. 1995, A\&A, 302, 587

Parenti, S., \& Vial, J.-C. 2007, A\&A, 469, 1109

Parenti, S., Schmieder, B., Heinzel, P., \& Golub, L. 2012, ApJ, 754, 66

Schmieder, B., Lin, Y., Heinzel, P., \& Schwartz, P. 2004, Sol. Phys., 221, 297

Schmieder, B., Gunár, S., Heinzel, P., \& Anzer, U. 2007, Sol. Phys., 241, 53

Schmieder, B., Chandra, R., Berlicki, A., \& Mein, P. 2010, A\&A, 514, A68

Schmit, D. J., \& Gibson, S. 2013, ApJ, 770, 35

Schmit, D. J., Gibson, S., Luna, M., Karpen, J., \& Innes, D. 2013, ApJ, 779, 156

Schühle, U. 2003, SUMER Data Cookbook, Published on internet at http:// www.mps.mpg.de/projects/soho/sumer/text/cookbook.html

Schwartz, P., Heinzel, P., Schmieder, B., \& Anzer, U. 2006, A\&A, 459, 651

Schwartz, P., Schmieder, B., Heinzel, P., \& Kotrč, P. 2012, Sol. Phys., 281, 707

Su, Y., \& van Ballegooijen, A. 2012, ApJ, 757, 168

Tsuneta, S., Ichimoto, K., Katsukawa, Y., et al. 2008, Sol. Phys., 249, 167

van Ballegooijen, A. A., \& Su, Y. 2014, in IAU Symp., 300, 127

Wang, H., Chae, J., Gurman, J. B., \& Kucera, T. A. 1998, Sol. Phys., 183, 91

Wiik, J. E., Dere, K., \& Schmieder, B. 1993, A\&A, 273, 267

Wilhelm, K., Curdt, W., Marsch, E., et al. 1995, Sol. Phys., 162, 189

Zirker, J. B., Engvold, O., \& Martin, S. F. 1998, Nature, 396, 440 


\section{Appendix A}
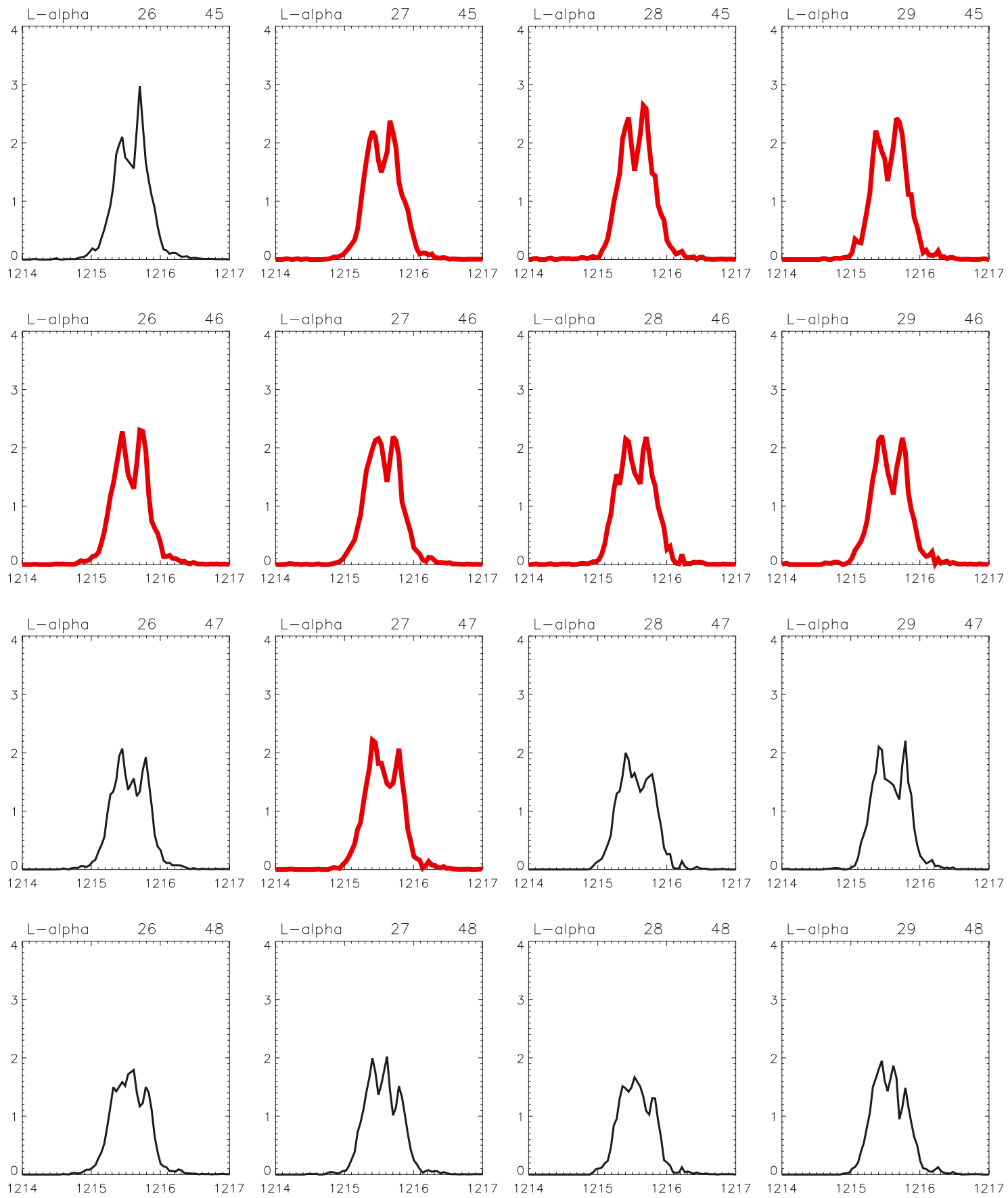

Fig. A.1. Small portion of the observed Lyman- $\alpha$ profiles from AREA1. The profiles selected for the analysis are marked in red. Numbers above each panel indicate the $x$ and $y$ positions in the SOHO/SUMER raster. The $x$-axis gives the wavelength in $\AA$, the $y$-axis the specific intensity in $10^{-8} \mathrm{erg} \mathrm{s}^{-1} \mathrm{~cm}^{-2} \mathrm{sr}^{-1} \mathrm{~Hz}^{-1}$. 
S. Gunár et al.: Magnetic field and radiative transfer modelling of a quiescent prominence
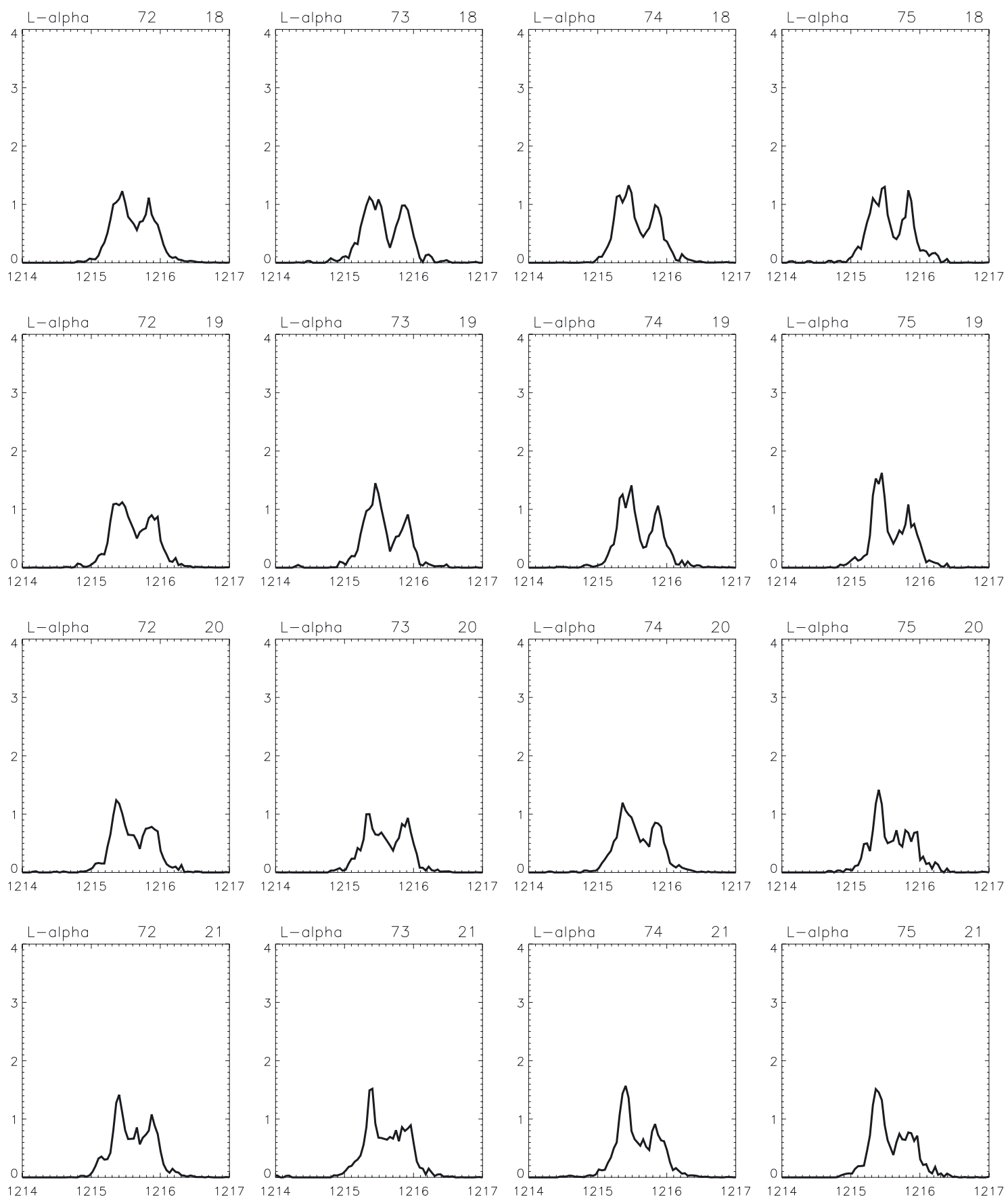

Fig. A.2. Small portion of the observed Lyman- $\alpha$ profiles from AREA2. Numbers above each panel indicate the $x$ and $y$ positions in the SOHO/SUMER raster. The $x$-axis gives the wavelength in $\AA$, the $y$-axis the specific intensity in $10^{-8} \mathrm{erg} \mathrm{s}^{-1} \mathrm{~cm}^{-2} \mathrm{sr}^{-1} \mathrm{~Hz}^{-1}$. 
A\&A 567, A123 (2014)
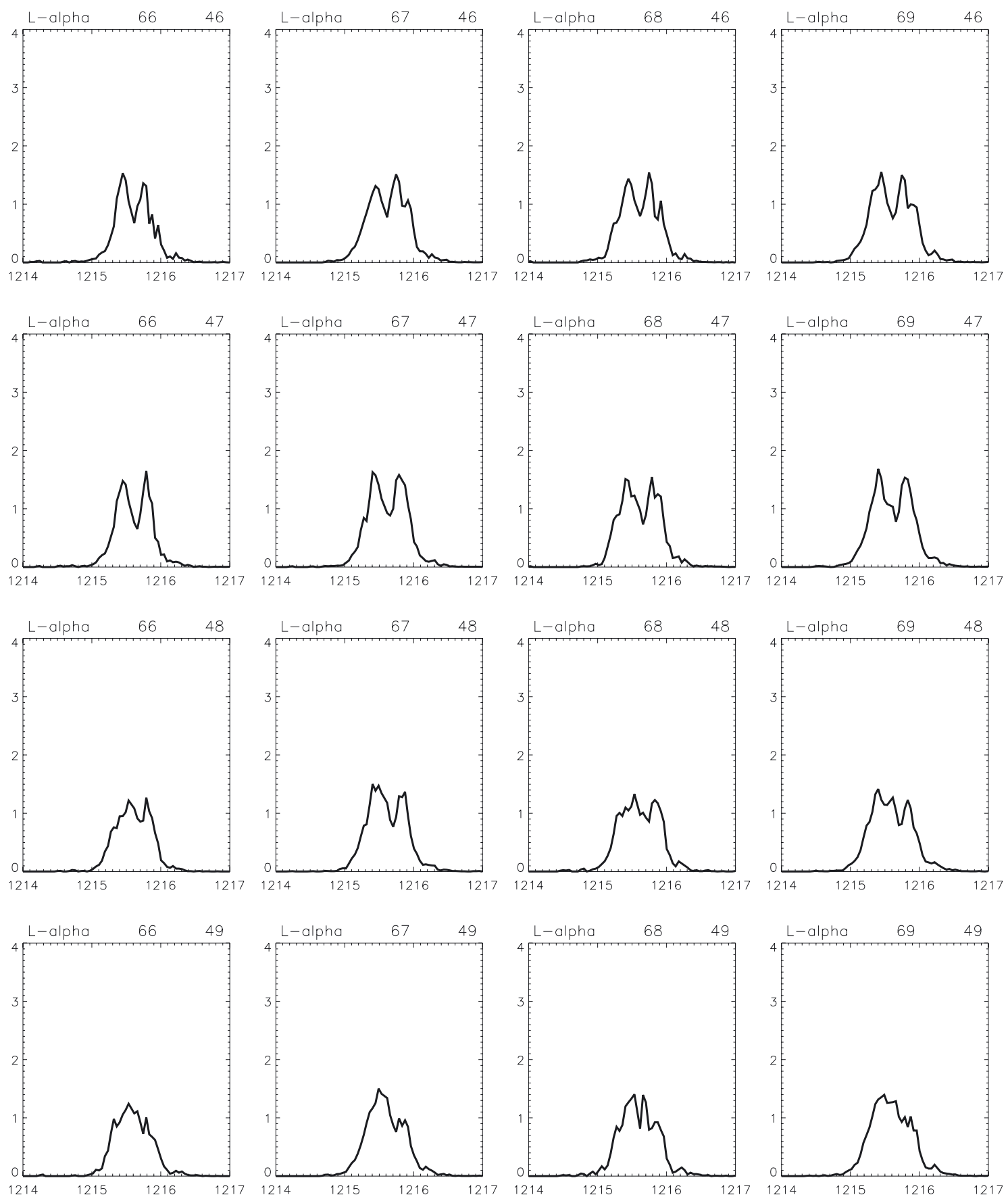

Fig. A.3. Small portion of the observed Lyman- $\alpha$ profiles from AREA3. Numbers above each panel indicate the $x$ and $y$ positions in the SOHO/SUMER raster. The $x$-axis gives the wavelength in $\AA$, the $y$-axis the specific intensity in $10^{-8} \mathrm{erg} \mathrm{s}^{-1} \mathrm{~cm}^{-2} \mathrm{sr}^{-1} \mathrm{~Hz}^{-1}$. 